\title{
3 Die AFGHANISCHE COMMUNITY IN ÖSTERREICH
}

\subsection{Einleitung}

Im Vergleich zu anderen europäischen Staaten (z.B. Deutschland oder Großbritannien), wo es bereits vor den kriegsbedingten Fluchtbewegungen seit langem bestehende und zahlenmäßig relativ große afghanische Communities gab (vgl. BRAAKMAN 2005), kam es in Österreich erst in den späten 1960er bzw. frühen 1970er Jahren zur Entstehung einer afghanischen Community. ${ }^{75}$ Diese geht im Wesentlichen auf den Zuzug einiger Dutzend, meist junger Afghanen (Frauen waren damals kaum darunter) zurück, die zum Studium nach Österreich kamen und meist in Wien lebten. Sie entstammten großteils dem urbanen Milieu Kabuls und gehörten zum überwiegenden Teil der paschtunischen oder tadschikischen Ethnie an. ${ }^{76}$

Erst mit dem Putsch der VDPA im April 1978 und den seither vonstattengehenden kriegerischen Auseinandersetzungen ist die Zahl der in Österreich dauerhaft ansässigen Afghan/inn/en sukzessive angestiegen und erreichte 2015 mit über 25.000 Asylwerber/ inne/n einen vorläufigen Höhepunkt. Mit Stichtag 1.1.2020 lebten laut Statistik Austria insgesamt 43.585 Personen mit afghanischer Staatsangehörigkeit sowie mehrere tausend inzwischen eingebürgerte Afghan/inn/en in Österreich. ${ }^{77}$ Die meisten Afghan/inn/en haben ihren Lebensmittelpunkt in Wien (rund 43\%) sowie in Oberösterreich (16\%), v.a. im urbanen Raum Linz und Wels. Die Steiermark (11\%) und Niederösterreich (10\%) stellen weitere Zentren der afghanischen Communities dar (Details vgl. unten).

Da der Großteil der heute in Österreich ansässigen Afghan/inn/en erst infolge der Fluchtmigration der letzten Jahre nach Österreich gekommen ist, soll zunächst ein kurzer Überblick über die Entwicklung der afghanischen Fluchtmigration gegeben werden und dann auf einzelne Aspekte der afghanischen Community (z.B. soziokulturelle Differenzierung der afghanischen Zuwander/er/innen, Vereine, regionale Verteilung etc.) näher eingegangen werden.

\footnotetext{
Bislang existieren keine Studien, die einen allgemeinen Überblick über die Geschichte der afghanischen Community in Österreich bieten würden. Einzelne in jüngerer Zeit erschienene Arbeiten behandeln meist Teilaspekte. Eine von Alam (2008) publizierte Arbeit liefert zwar statistisches Zahlenmaterial zu afghanischen Flüchtlingen der 1980er und 1990er Jahre, bietet aber ansonsten keinerlei Hintergrundinformationen zur afghanischen Community, ähnlich der 2011 veröffentlichte Aufsatz von POTKANSKI.

76 Persönliche Erinnerungen von G. RasulY-PALECZEK, die seit Mitte der 1970er Jahre Kontakte zur afghanischen Community in Wien hatte.

77 Laut Angaben der Statistik Austria wurde zwischen 1981 und 2016 insgesamt 3.504 Afghan/ inn/en die österreichische Staatsbürgerschaft verliehen.
} 


\subsection{Die afghanische Fluchtmigration nach Österreich}

Was die Fluchtmigration afghanischer Staatsbürger/innen angeht, so lassen sich auch hier vier Phasen unterscheiden (vgl. oben):

1) Vom kommunistischen Putsch bis zum Sieg der Mujaheddin (1978-1992)

2) Während der Herrschaft der Mujaheddin (1992-1996)

3) Während der Taliban-Herrschaft (1996-2001)

4) Post-Taliban-Ära (ab 2002)

Während anfänglich nur sehr wenige Afghan/inn/en Zuflucht in Österreich suchten, wurde das Land in den letzten Jahren zu einem der Hauptzielländer afghanischer Asylwerber/innen in Europa (vgl. Details oben). Die Entwicklung der Asylantragszahlen verlief nicht kontinuierlich, sondern spiegelt sehr deutlich die politischen Entwicklungen in Afghanistan sowie - vor allem was die Post-Taliban-Ära anbelangt - auch die Flüchtlingspolitik der beiden „klassischen Aufnahmeländer“ afghanischer Flüchtlinge, Iran und Pakistan, wider. Auch der soziokulturelle Background der in Österreich asylsuchenden Personen hat sich seit dem Beginn der Fluchtmigration im Jahr 1978 deutlich verändert. Im Unterschied zu früheren Asylwerber/inne/n, die meist der gebildeten, urbanen Mittel- und Oberschicht angehörten, weisen die nun kommenden Flüchtlinge eine erhebliche Heterogenität in Bezug auf ihren sozioökonomischen und bildungsmäßigen Hintergrund auf.

\subsubsection{Erste größere Fluchtmigration nach Österreich $(1978-1992)^{78}$}

Die ersten afghanischen Flüchtlinge kamen bereits kurz nach dem Putsch der kommunistischen Partei (April 1978) nach Österreich. Im Vergleich zu späteren Perioden waren die Zahlen allerdings äußerst bescheiden. Laut Angaben des Statistischen Handbuchs der Republik Österreich (zit. nach KAFÖ 1984, p. 180) flohen 1978 drei Personen und 1979 achtzehn Personen nach Österreich. Nach dem Einmarsch der sowjetischen Truppen (Dez. 1979) stieg ihre Zahl an und erreichte 1982 mit 68 und im Folgejahr mit 161 Personen einen ersten Höhepunkt (BELler 2013, p. 26, Tab. 1) (vgl. Abb. 6).

78 Zur Fluchtmigration der späten 1970er und frühen 1980er Jahre liegen keine detaillierten Informationen vor. Laut Auskunft eines Exmitarbeiters von Statistik Austria (E-Mail an J. KOHLBACHER vom 7. Februar 2018) wurden in den 1970er Jahren für kleine Bevölkerungsgruppen, wie z.B. die afghanische Community, keine Wanderungsstatistiken auf Herkunftsbasis erstellt. Laut der Medien-Servicestelle Neue ÖsterReicher/InNEN gibt es erst seit 1980 öffentlich zugängliche statistische Aufzeichnungen des BM.I zu Asylwerber/inne/n (siehe MEDIEN-SERVICESTELle Neue ÖsterReicher/InNEn 2016a). Die im Folgenden genannten Informationen basieren im Wesentlichen auf persönlichen Erinnerungen von G. RASULY-PALECZEK, die damals gemeinsam mit anderen Afghan/Inn/en und Österreicher/inne/n im Rahmen des ,,Sozialistischen Afghanistan Komitees“ in die ehrenamtliche Betreuung von Asylwerber/inne/n aus Afghanistan involviert war. 
Abb. 6: Asylwerber/innen aus Afghanistan in Österreich vom kommunistischen Putsch bis zum Sieg der Mujaheddin (1978-1992)

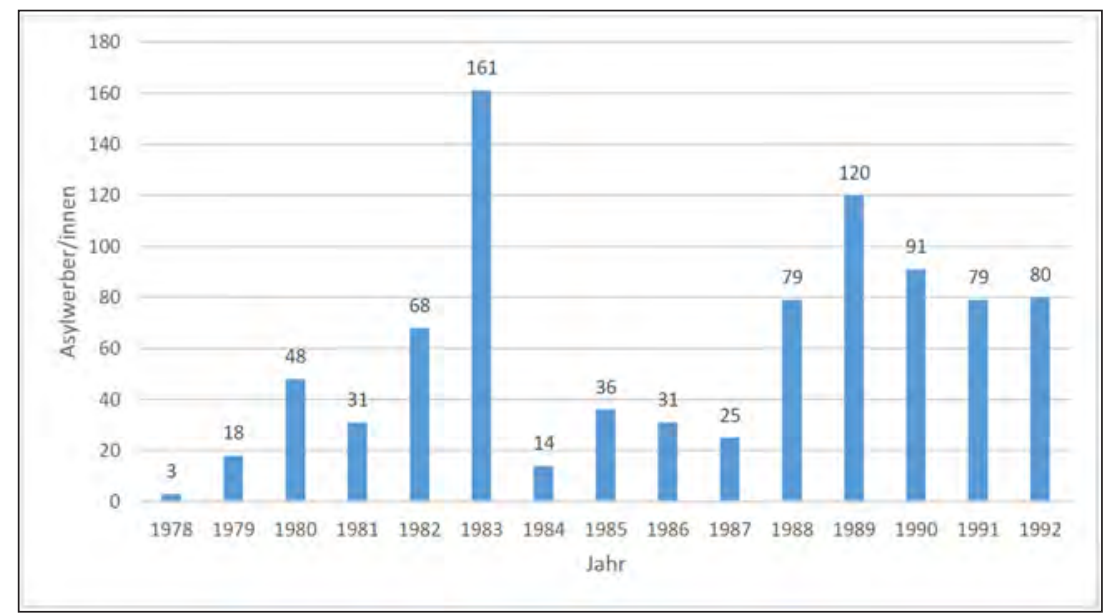

Quellen: Daten des Statistischen Handbuchs für die Republik Österreich, zit. nach KAFÖ (1984, p. 180) und BeLler (2013, p. 26, Tab. 1).

Unter den damals nach Österreich gekommenen Afghan/inn/en (fast ausschließlich Männern) befanden sich einerseits Mitglieder und Funktionäre der Khalq-Fraktion der VDPA (Volksdemokratische Partei Afghanistans), die im Zuge von Fraktionskämpfen innerhalb der VDPA in Ungnade gefallen waren ${ }^{79}$ und andererseits um eine größere Gruppe von jungen Männern (darunter viele Hazara, aber auch einige aus dem Pandschir-Tal stammende Tadschiken), die über einen Zwischenstopp im Iran nach Österreich gelangten. ${ }^{80}$ Die meisten von ihnen erhielten in der Folge Asyl in Österreich, begannen eine Berufsausbildung und/oder ein Studium und heirateten später teilweise afghanische Frauen, die sie aus Afghanistan, Iran oder Pakistan nach Österreich brachten. Einzelne besser ausgebildete Afghan/inn/en migrierten von Österreich in die USA oder nach Kanada. ${ }^{81} \mathrm{Zu}$ den in den 1980er Jahren nach Österreich kommenden Asylwerber/inne/n zählten auch Familienangehörige von Personen, die bereits seit

79 Aus den parteiinternen Streitigkeiten ging der Parcham-Flügel als Sieger hervor. Angeführt von Babrak Karmal regierten die Parchamis unterstützt von den sowjetischen Invasionstruppen in den folgenden Jahren das Land. Zahlreiche Khaliqs flohen damals in den Westen oder in die Sowjetunion.

80 Rund 100 junge Männer kamen um das Jahr 1983 nach Österreich, darunter befanden sich schon damals viele, die nur eine rudimentäre Schulbildung aufwiesen. Für sie wurden in Österreich später Alphabetisierungs- und Berufsbildungskurse, z.B. in Mödling, organisiert (persönliche Erinnerung G. RASULY-PALECZEK).

81 Einige Afghanen konnten nach erfolgreichen Interviews in den Botschaften der USA und Kanadas nach Nordamerika weitermigrieren, wobei beide Staaten sehr selektiv vorgingen und nur Personen mit guten Schul- und Berufsausbildungen eine Einwanderung ermöglichten (persönliche Erinnerung G. RaSuly-PALECZEK). 
den 1960er und 1970er Jahren im Land lebten und nun nahe Familienangehörige (z.B. Eltern, Geschwister) nach Österreich brachten und ihnen bei den Asylverfahren behilflich waren.

Nach 1983 ging die Zahl der Asylanträge afghanischer Staatsbürger/innen zunächst deutlich zurück (vgl. Abb. 6), stieg Ende der 1980er Jahre jedoch wieder an (1988 79 Personen und 1989 - im Jahr des Abzugs der sowjetischen Invasionstruppen - 120 Personen). Unter den damals nach Österreich geflohenen Personen befanden sich v.a. Funktionsträger/innen des kommunistischen Regimes oder Sympathisant/inn/en desselben.

\subsubsection{Während der Mujaheddin-Herrschaft (1992-1996)}

Mit der Machtergreifung der Mujaheddin im April 1992 begannen die Asylwerber/ innenzahlen ab 1993 (vgl. Abb. 7) neuerlich zu steigen und erreichten mit der Eroberung Kabuls durch die Taliban im Jahr 1996 mit 766 Asylanträgen einen ersten Spitzenwert. Bei den meisten damals nach Österreich geflohenen Personen handelte es sich ebenfalls primär um solche, die während der Herrschaft des kommunistischen Regimes hohe staatliche Ämter bekleidet hatten oder in der Verwaltung des Landes tätig gewesen waren, daneben aber auch um berufstätige Frauen, die sich durch die Mujaheddin-Regierung unter Druck gesetzt fühlten. Erstmals floh nun eine größere Zahl von allein reisenden Frauen und Familien nach Österreich.

Abb. 7: Asylwerber/innen aus Afghanistan in Österreich während der Herrschaft der Mujaheddin (1992-1996) und der Taliban-Ära (1996-2001)

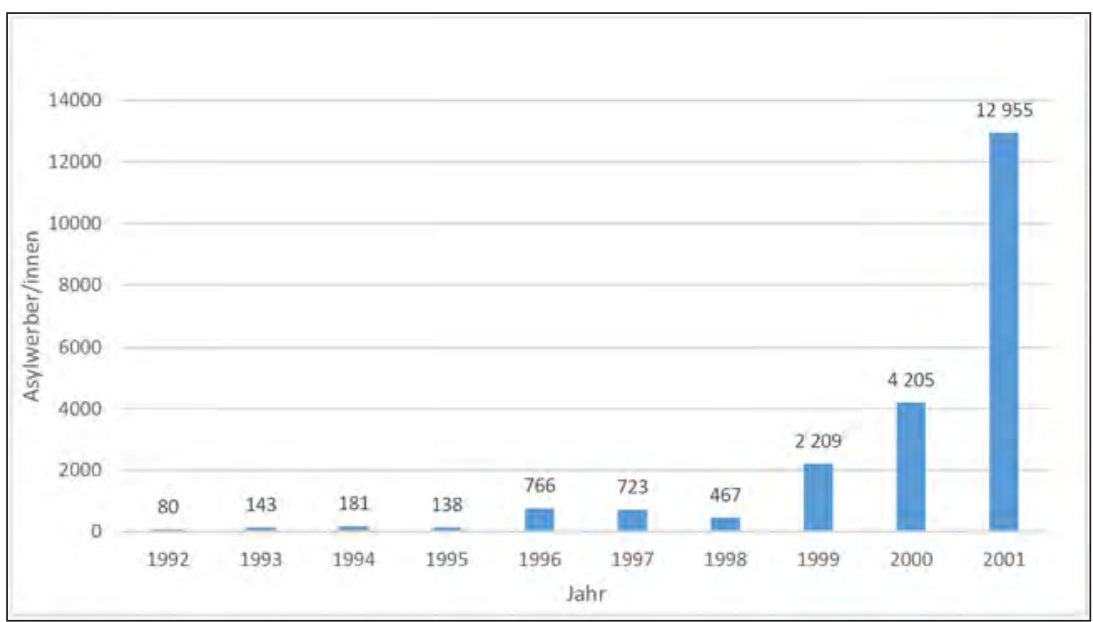

Quellen: Daten des UNHCR zit. nach Alam (2008, p. 18, Tab. 4) und Beller (2013, p. 28 und 30, Tab. 3). 


\subsubsection{Während der Taliban-Herrschaft (1996-2001)}

Während der Herrschaft der Taliban gingen die Asylanträge 1997 und 1998 zunächst kurzfristig zurück, stiegen aber ab 1999 erneut an. 2001 wurde mit 12.955 Anträgen ein erster Rekordwert erreicht (vgl. Abb. 7).

Warum gerade 2001 so viele Afghan/inn/en nach Österreich kamen und welchen soziokulturellen Background sie hatten, geht aus den zur Verfügung stehenden Quellen nicht hervor. Es liegt die Vermutung nahe, dass es sich dabei v.a. um Angehörige der afghanischen Schiiten (v.a. Hazara) handelte, die von den Taliban besonders verfolgt wurden (z.B. Massaker 1998 in Mazar-e Sharif) sowie um Angehörige der gebildeten Mittelklasse, die wegen der restriktiven Politik der Taliban (z.B. Bildungs- und Berufsverbot für Frauen flohen). Unter den nach Österreich gekommenen Personen könnten aber auch ehemalige Mitglieder der VDPA gewesen sein, die zunächst in den noch nicht von den Taliban kontrollierten Gebieten Nordafghanistans Schutz gesucht hatten und mit der Einnahme dieser Region durch die Taliban 1998 einen neuen Zufluchtsort suchen mussten.

\subsubsection{Während der Post-Taliban-Ära (seit 2001)}

Mit dem Zusammenbruch des Taliban-Regimes Ende 2001/Anfang 2002 gingen die Zahlen sukzessive zurück und erreichten 2006 mit nur 699 Asylanträgen einen Tiefstwert. Erst die sich ab 2009 zunehmend verschlechternde Sicherheitslage in Afghanistan und die immer schwieriger werdenden Lebensbedingungen afghanischer Flüchtlinge im Iran, in Pakistan und in der Türkei ließen die Zahl der Asylanträge neuerlich markant ansteigen (vgl. Abb. 8 und Abb. 9).

Abb. 8: Asylwerber/innen aus Afghanistan in Österreich vom Sturz der Taliban bis heute (2001-2019)

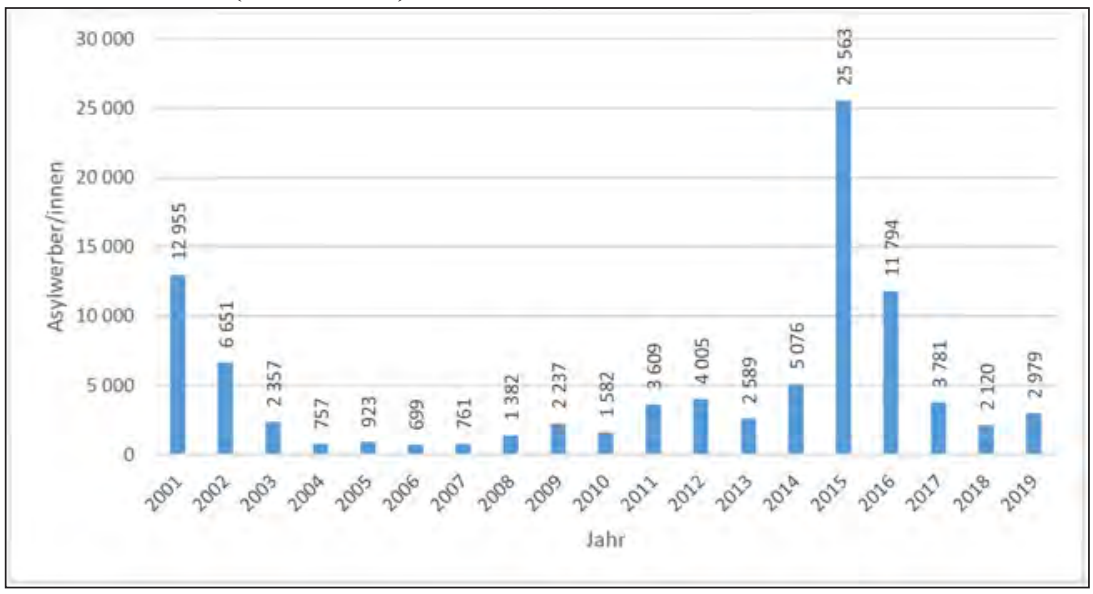

Quellen: Statistik-Austria-Daten zit. nach Alam (2008, p. 19, Tab. 5); Medien-Servicestelle NEUE ÖSTERREICHER/INNEN (2016b, basierend auf BM.I-Daten) sowie BM.I Asylstatistik 2014, 2015, 2016, 2017, 2018 und 2019. 
Abb. 9: Afghanische Asylwerber/innen in Österreich im Vergleich (2001-2019)

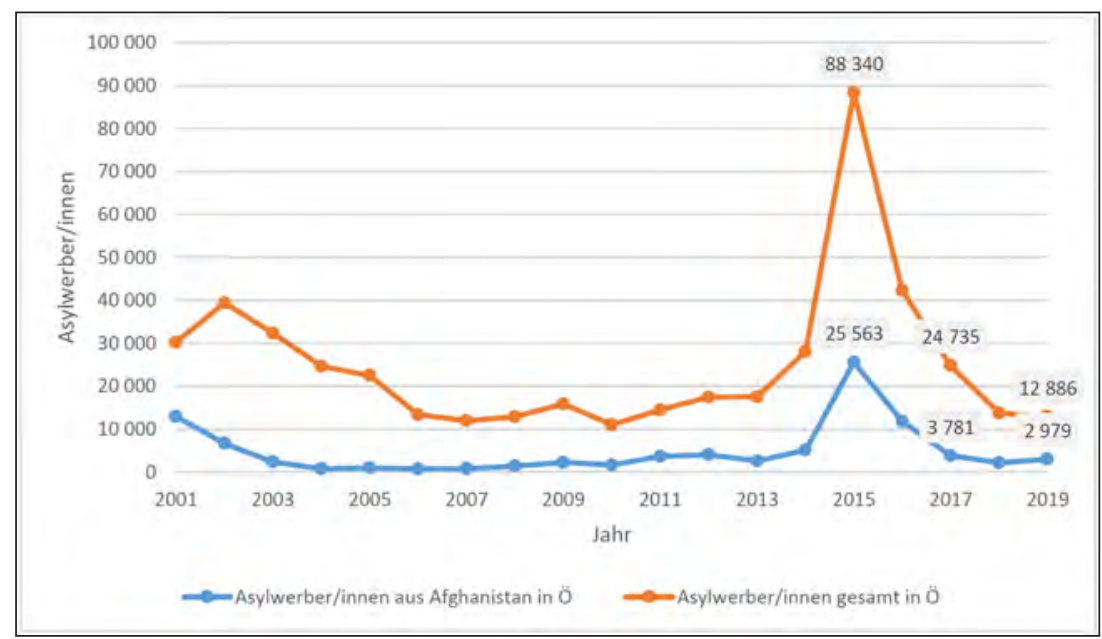

Quellen: Zusammengestellt nach RABL (2019, basierend auf BM.I Daten), Statistik-Austria-Daten zit. nach Alam (2008, p. 19, Tab. 5); Medien-Servicestelle Neue ÖSTERREICHER/INNEN (2016b, basierend auf BM.I-Daten) sowie BM.I Asylstatistik 2014, 2015, 2016, 2017, 2018 und 2019.

Ein absoluter Spitzenwert wurde 2015 erreicht, als insgesamt 25.563 Afghan/inn/ en ( $=29 \%$ aller 88.340 Asylantragsteller/innen) um Schutz baten. Sie lagen damit noch vor den Syrer/inne/n (in summa 24.547 Personen $=28 \%$ aller Asylwerber/innen) und den Iraker/inne/n (13.633 Personen $=15 \%)$. Seit Mitte 2016 sind die Zahlen afghanischer Asylanträge rückläufig. ${ }^{82}$ Bis Ende 2016 wurden nur mehr 11.794 und 2017 lediglich 3.781 Anträge gestellt (zum Vergleich stammten 7.356 also 30\% der insgesamt 24.735 Anträge von Syrer/inne/n). 2018 stellten 2.120 Afghan/inn/en (=15\% aller Asylwerber/ innen) einen Antrag in Österreich. 2019 stieg die Zahl mit 2.979 (=23\% aller Anträge) wieder leicht an.

Was den sozioökonomischen und ethnolinguistischen Background der in den letzten Jahren nach Österreich gekommenen Afghan/inn/en angeht, so ist dieser im Unterschied zu den Asylwerber/inne/n früherer Zuwanderungsphasen sehr heterogen.

Während in den 1980er und 1990er Jahren fast ausschließlich Personen kamen, die der urbanen Ober- und Mittelschicht angehörten und meist eine gute Schul- und Berufsausbildung hatten, stammen viele der rezenten Asylsuchenden aus der urbanen Unterschicht oder aus dem ländlichen Raum und weisen oft keine oder nur eine rudimentäre Schul- und Berufsausbildung auf. Zahlreiche der nun nach Österreich kommenden 82 Insgesamt verzeichnete Österreich zwischen 2016 und 2018 einen deutlichen Rückgang der Antragszahlen: von 2015 auf $2016-52 \%$, von 2016 auf 2017 um -41\%, von 2017 auf 2018 um -44\% (BM.I Asylstatistik 2014, 2015, 2016, 2017 und 2018). 
Tab. 1: Erstanträge von Asylwerber/inne/n nach Staatsangehörigkeit (2014-2019)

\begin{tabular}{|c|c|c|c|c|c|c|}
\hline & \multicolumn{2}{|c|}{2014} & \multicolumn{2}{|c|}{2015} & \multicolumn{2}{|c|}{2016} \\
\hline & absolut & in \% & absolut & in \% & absolut & in \% \\
\hline Afghanistan & 5.076 & 18,08 & 25.563 & 28,93 & 11.794 & 27,89 \\
\hline Syrien & 7.730 & 27,54 & 24.547 & 27,78 & 8.773 & 20,74 \\
\hline Irak & 1.105 & 3,90 & 13.633 & 15,43 & 2.862 & 6,76 \\
\hline Summe Anträge & 28.064 & & 88.340 & & 42.285 & \\
\hline & \multicolumn{2}{|c|}{2017} & \multicolumn{2}{|c|}{2018} & \multicolumn{2}{|c|}{2019} \\
\hline & absolut & in \% & absolut & in $\%$ & absolut & in \% \\
\hline Afghanistan & 3.781 & 15,28 & 2.120 & 15,42 & 2.979 & 23,11 \\
\hline Syrien & 7.356 & 29,75 & 3.329 & 24,21 & 2.708 & 21,01 \\
\hline Irak & 1.403 & 5,60 & 762 & 5,54 & 729 & 5,60 \\
\hline Summe Anträge & 24.735 & & 13.746 & & 12.886 & \\
\hline
\end{tabular}

Quellen: BM.I Asylstatistik 2014, 2015, 2016, 2017, 2018 und 2019.

Abb. 10: Erstanträge von Asylwerber/inne/n nach Staatsangehörigkeit (2014-2019)

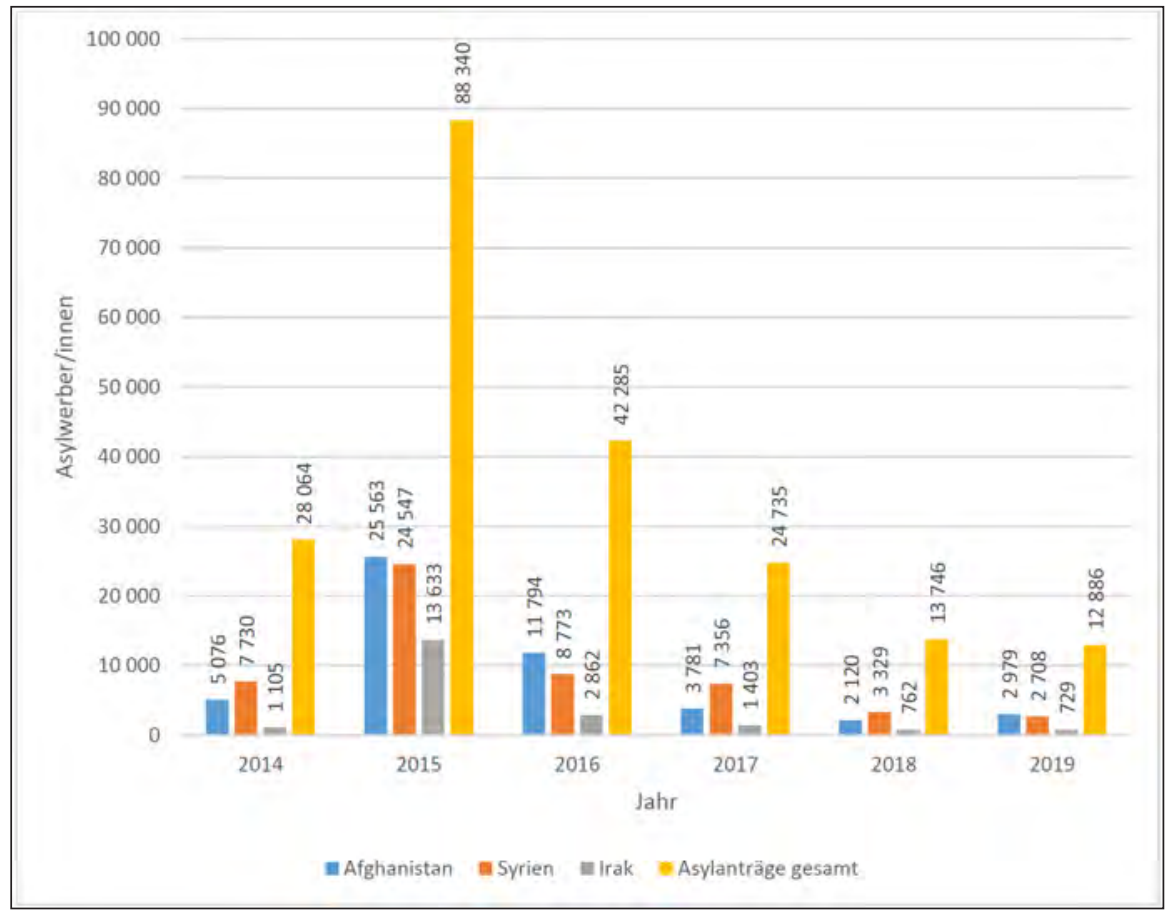

Quellen: BM.I Asylstatistik 2014, 2015, 2016, 2017, 2018 und 2019. 
Afghan/inn/en haben eine lange Fluchtgeschichte hinter sich. Sie waren zuvor IDPs (Internally Displaced Persons) oder haben länger in den beiden Nachbarstaaten Iran und Pakistan gelebt bzw. wurden sogar dort geboren. Auch bezüglich ihres ethnolinguistischen Backgrounds unterscheiden sich die heutigen afghanischen Asylsuchenden von den früher geflohenen. Waren es anfänglich v.a. sunnitische Dari-Sprechende (z.B. Tadschiken, Kabuli und iranisierte Paschtunen ${ }^{83}$ sowie einige schiitische Hazara), so befinden sich unter den rezenten afghanischen Asylwerber/inne/n Angehörige aller ethnolinguistischen Gruppen (z.B. Turkmenen, Uzbeken etc.), wobei insbesondere die Hazara einen sehr hohen Anteil stellen dürften (genaue Angaben hierzu fehlen allerdings).

Ein weiteres Charakteristikum der rezenten afghanischen Asylwerber/innen ist der hohe Anteil von jungen Männern und männlichen UMF. Im Unterschied zu Flüchtlingen aus Syrien ist der Anteil allein fliehender Afghan/inn/en oder ganzer Familien gering. Die Gründe hier dürften vor allem in den gefährlicheren und längeren Fluchtwegen sowie dem häufigen Fehlen entsprechender finanzieller Mittel liegen.

Unter den nach Österreich kommenden Asylwerber/inne/n gibt es auch zahlreiche UMF. Ihre Zahl ist in den letzten Jahren deutlich angestiegen. Allein zwischen 2014 und 2015 von 1.976 auf 8.277 Personen, damit stellten sie 2015 fast 10\% aller Asylwerber/ innen, wobei 5.609 UMF (=68\% aller UMF) einen afghanischen Hintergrund hatten.

Tab. 2: Entwicklung der Asylanträge afghanischer UMF in Österreich (2005-2019)

\begin{tabular}{|c|r|r|r|}
\hline \multirow{2}{*}{ Jahr } & \multicolumn{2}{|c|}{$\begin{array}{c}\text { UMF } \\
\text { Anträge gesamt }\end{array}$} & \multicolumn{2}{|c|}{$\begin{array}{c}\text { Anträge } \\
\text { afghanische UMF } \\
\text { absolut }\end{array}$} \\
\hline 2005 & 881 & 93 & in \% \\
\hline 2006 & 488 & 46 & 10,56 \\
\hline 2007 & 582 & 100 & 17,18 \\
\hline 2008 & 874 & 242 & 27,68 \\
\hline 2009 & 1.185 & 431 & 36,37 \\
\hline 2010 & 934 & 297 & 31,79 \\
\hline 2011 & 1.346 & 755 & 56,09 \\
\hline 2012 & 1.781 & 1.035 & 58,11 \\
\hline 2013 & 1.187 & 437 & 36,81 \\
\hline 2014 & 1.976 & 1.201 & 60,77 \\
\hline 2015 & 8.277 & 5.609 & 67,76 \\
\hline 2016 & 3.900 & 2.446 & 62,71 \\
\hline 2017 & 1.352 & 694 & 51,33 \\
\hline 2018 & 390 & 163 & 41,79 \\
\hline 2019 & 859 & 642 & 74,73 \\
\hline
\end{tabular}

Quellen: Medien-Servicestelle Neue Österreicher/InNen (2016b) und BM.I Asylstatistik 2015, 2016, 2017, 2018 und 2019.

83 Paschtunen, die in den städtischen Zentren (z.B. in Kabul) leben, haben häufig ihre Sprache - das Paschtu - zugunsten des Dari aufgegeben. Die Bewohner/innen Kabuls werden in Afghanistan unabhängig von ihrem ethnolinguistischen Background als Kabuli bezeichnet. 
Abb. 11: Entwicklung der Asylanträge afghanischer UMF in Österreich (2005-2019)

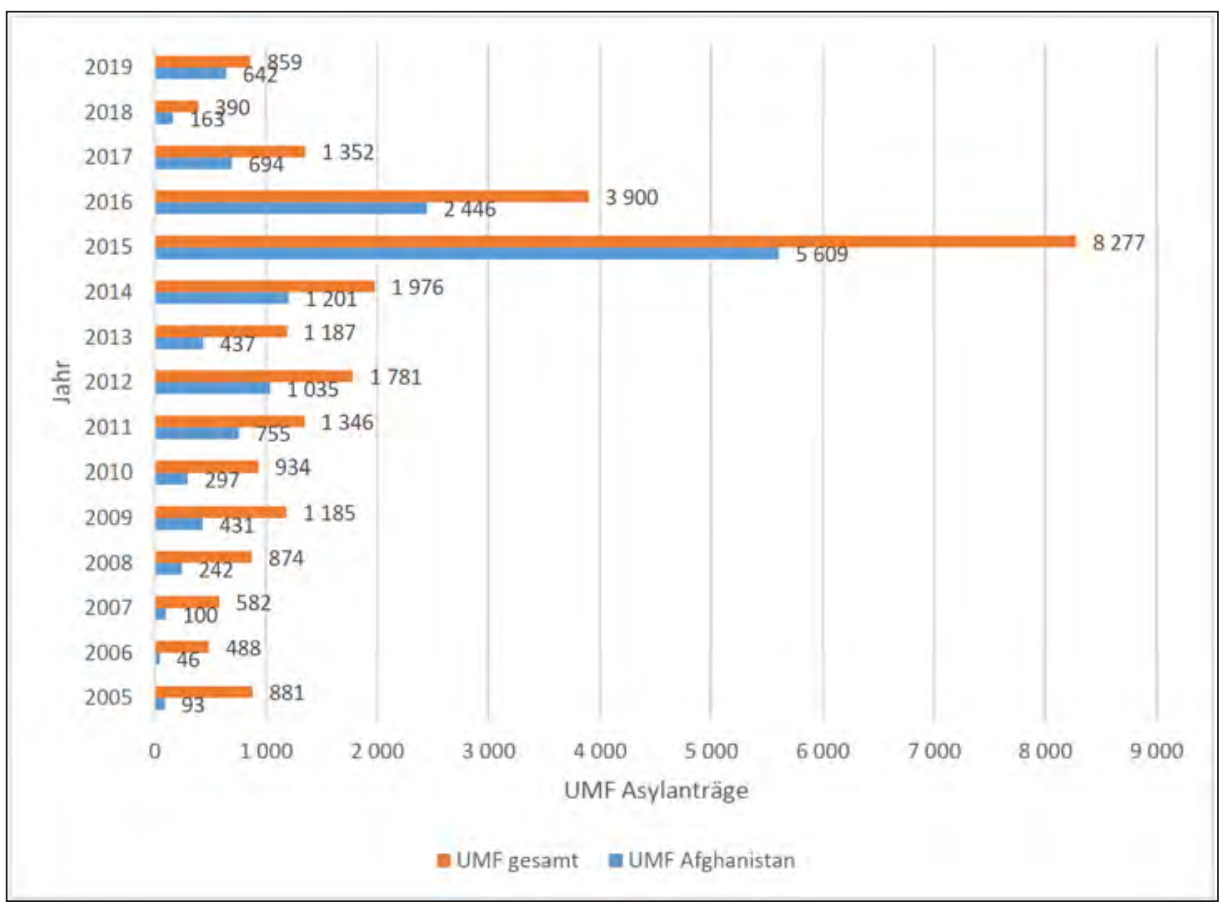

Quellen: Medien-Servicestelle Neue Österreicher/innen (2016b) und BM.I Asylstatistik 2015, 2016, 2017, 2018 und 2019.

Insbesondere die Zahl von UMF aus Afghanistan hat sich zwischen 2014 und 2015 rasant erhöht. 2014 stellten sie mit 1.201 Kindern und Jugendlichen 61\% aller UMF, 2015 kamen bereits 5.609 afghanischen UMF (=68\% aller UMF). Im Vergleich dazu stammten 2015 ,nur“ 1.134 Kinder und Jugendliche (=14\%) aus Syrien und lediglich $366(=4 \%)$ aus dem Irak.

Seit 2016 sind die UMF-Zahlen rückläufig, dennoch stellten UMF mit afghanischem Background (viele kommen eigentlich aus dem Iran und gehören meist der Gruppe der Hazara an) 2016 rund 63\% aller UMF und 2018 mit 163 Personen immerhin noch 42\% aller UMF. 2019 stieg die Zahl der UMF wieder an. Afghanische UMF stellten hier mit 642 Personen $(=75 \%)$ den größten Anteil.

Auch ein Großteil der UMF in der Altersgruppe unter 14 Jahren weist einen afghanischen Hintergrund auf. Während 201431 der insgesamt 119 registrierten UMF unter 14 Jahren aus Syrien stammten, hatten 62 (=52\% aller UMF) einen afghanischen Hintergrund. 2015 stellten sie mit 427 Kindern einen Anteil von 58\% aller UMF 
Tab. 3: Veränderung der UMF-Zahlen (unter 14 sowie von 14 bis 18 Jahren) aus Afghanistan, Syrien und Irak in Österreich (2014-2019)

\begin{tabular}{|c|c|c|c|c|c|c|}
\hline & \multicolumn{2}{|c|}{2014} & \multicolumn{2}{|c|}{2015} & \multicolumn{2}{|c|}{2016} \\
\hline & absolut & in $\%$ & absolut & in $\%$ & absolut & in $\%$ \\
\hline Afghanistan & 1.201 & 60,77 & 5.609 & 67,76 & 2.446 & 62,71 \\
\hline Syrien & 257 & 13,00 & 1.134 & 13,70 & 130 & 3,33 \\
\hline Irak & 29 & 1,46 & 366 & 4,42 & 84 & 2,51 \\
\hline Summe Anträge & 1.976 & & 8.277 & & 3.900 & \\
\hline & \multicolumn{2}{|c|}{2017} & \multicolumn{2}{|c|}{2018} & \multicolumn{2}{|c|}{2019} \\
\hline & absolut & in \% & absolut & in \% & absolut & in \% \\
\hline Afghanistan & 694 & 51,33 & 163 & 41,79 & 642 & 74,73 \\
\hline Syrien & 59 & 4,36 & 38 & 9,74 & 54 & 6,28 \\
\hline Irak & 55 & 4,06 & 20 & 5,12 & 14 & 1,62 \\
\hline Summe Anträge & 1.352 & & 390 & & 859 & \\
\hline
\end{tabular}

Quellen: BM.I Asylstatistik 2014, 2015, 2016, 2017, 2018 und 2019.

Abb. 12: Veränderung der UMF-Zahlen aus Afghanistan, Syrien und Irak in Österreich (2014-2019)

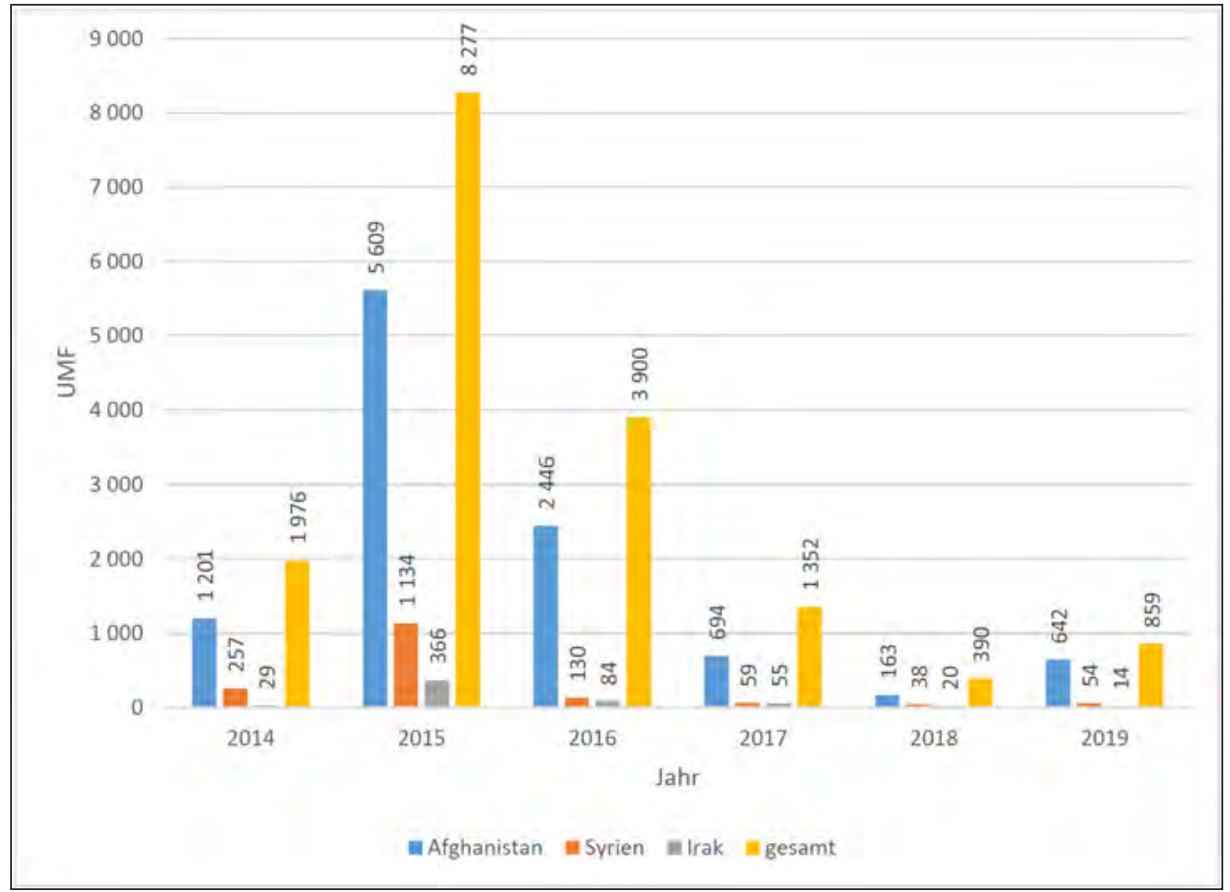

Quellen: BM.I Asylstatistik 2014, 2015, 2016, 2017, 2018 und 2019. 
in dieser Altersgruppe. Seither sind die UMF-Zahlen zwar rückläufig - 2016 stellten insgesamt 372 und 2017 nur mehr 143 Kinder einen Asylantrag -, dennoch entfielen auf junge Afghan/inn/en mit 285 (=77\% aller Anträge) im Jahr 2016 und 104 Kindern (= 73\%) im Jahr 2017 überproportional hohe Anteile.

Tab. 4: Asylsuchende UMF aus Afghanistan, Syrien und Irak nach Alter der Antragsteller/innen (2014-2019)

\begin{tabular}{|c|c|c|c|c|c|c|c|c|}
\hline & \multicolumn{4}{|c|}{2014} & \multicolumn{4}{|c|}{2015} \\
\hline & $\begin{array}{c}\text { unter } 14 \\
\text { Jahren }\end{array}$ & $\begin{array}{c}\% \text { davon } \\
\text { unter } \\
14 \mathrm{~J} .\end{array}$ & $\begin{array}{c}\text { 14-18 } \\
\text { Jahre }\end{array}$ & gesamt & $\begin{array}{c}\text { unter } 14 \\
\text { Jahren }\end{array}$ & $\begin{array}{c}\% \text { davon } \\
\text { unter } \\
14 \mathrm{~J} .\end{array}$ & $\begin{array}{c}\text { 14-18 } \\
\text { Jahre }\end{array}$ & gesamt \\
\hline Afghanistan & 62 & 52,10 & 1.139 & 1.201 & 427 & 57,46 & 5.182 & 5.609 \\
\hline Syrien & 31 & 26,00 & 226 & 257 & 182 & 24,49 & 952 & 1.134 \\
\hline Irak & 2 & 1,68 & 27 & 29 & 64 & 8,60 & 302 & 366 \\
\hline \multirow[t]{3}{*}{ Summe Anträge } & 119 & & 1.857 & 1.976 & 743 & & 7.534 & 8.277 \\
\hline & \multicolumn{4}{|c|}{2016} & \multicolumn{4}{|c|}{2017} \\
\hline & $\begin{array}{c}\text { unter } 14 \\
\text { Jahren }\end{array}$ & $\begin{array}{c}\% \text { davon } \\
\text { unter } \\
14 \mathrm{~J} .\end{array}$ & $\begin{array}{c}14-18 \\
\text { Jahre }\end{array}$ & gesamt & $\begin{array}{c}\text { unter } 14 \\
\text { Jahren }\end{array}$ & $\begin{array}{c}\% \text { davon } \\
\text { unter } \\
14 \mathrm{~J} .\end{array}$ & $\begin{array}{c}14-18 \\
\text { Jahre }\end{array}$ & gesamt \\
\hline Afghanistan & 285 & 76,61 & 2.161 & 2.446 & 104 & 72,72 & 590 & 694 \\
\hline Syrien & 22 & 5,91 & 108 & 130 & 6 & 4,19 & 53 & 59 \\
\hline Irak & 20 & 5,37 & 64 & 84 & 7 & 4,89 & 48 & 55 \\
\hline \multirow[t]{3}{*}{ Summe Anträge } & 372 & & 3.528 & 3.900 & 143 & & 1.209 & 1.352 \\
\hline & \multicolumn{4}{|c|}{2018} & \multicolumn{4}{|c|}{2019} \\
\hline & $\begin{array}{c}\text { unter } 14 \\
\text { Jahren }\end{array}$ & $\begin{array}{c}\% \text { davon } \\
\text { unter } \\
14 \mathrm{~J} .\end{array}$ & $\begin{array}{c}14-18 \\
\text { Jahre }\end{array}$ & gesamt & $\begin{array}{c}\text { unter } 14 \\
\text { Jahren }\end{array}$ & $\begin{array}{c}\% \text { davon } \\
\text { unter } \\
14 \mathrm{~J} .\end{array}$ & $\begin{array}{c}\text { 14-18 } \\
\text { Jahre }\end{array}$ & gesamt \\
\hline Afghanistan & 20 & 40,81 & 143 & 163 & 27 & 50,94 & 615 & 642 \\
\hline Syrien & 9 & 18,36 & 29 & 38 & 12 & 22,64 & 42 & 54 \\
\hline Irak & 7 & 14,28 & 13 & 20 & 1 & 1,89 & 13 & 14 \\
\hline Summe Anträge & 49 & & 341 & 390 & 53 & & 806 & 859 \\
\hline
\end{tabular}

Quellen: BM.I Asylstatistik 2014, 2015, 2016, 2017, 2018 und 2019.

Was die Zuerkennung einer positiven Asylentscheidung angeht, so lassen sich erhebliche Schwankungen erkennen. Während 2006 64\% aller afghanischen Asylwerber/ innen einen positiven Bescheid erhielten, war dies 2010 nur bei 34\% der Antragsteller/ innen der Fall. Danach stieg der Anteil der positiven Entscheidungen wieder an und erreichte 2014 mit 53\% einen neuen Höchstwert. Seither sind die positiven Entscheidungen wieder rückläufig. Mit nur 25\% im Jahre 2016 lag ein seit 2008 nicht mehr verzeichneter Tiefstwert vor. 2017 stiegen die Positiventscheidungen wieder auf $47 \%$ an und erreichten 2018 50\%, um danach wieder auf 46\% zurückzugehen (vgl. Abb. 13). 
Abb. 13: Anerkennungsquoten afghanischer Asylwerber/innen (2006-2019)

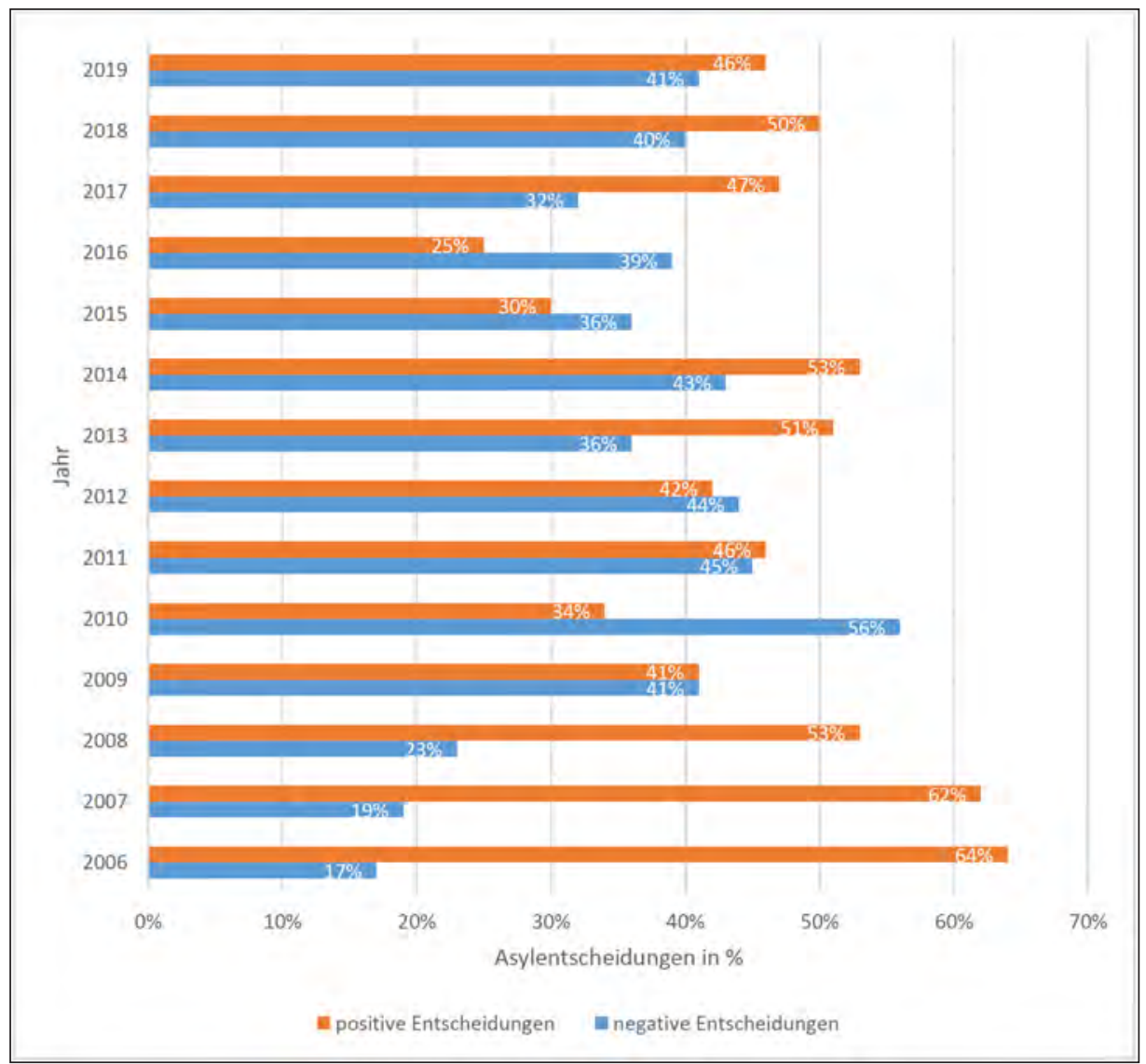

Quellen: Medien-Servicestelle Neue Österreicher/InNen (2016b) und BM.I Asylstatistik 2015, 2016, 2017, 2018 und 2019.

Vergleicht man die Anerkennungsquoten afghanischer Asylwerber/innen mit anderen Gruppen von Antragsteller/inne/n (z.B. Syrer/innen), so zeigt sich bei den Afghan/inn/en eine deutlich niedrigere Anerkennungsquote als bei Geflüchteten aus Syrien. Bei Letzteren lag sie 2014 bei 88\%, 2015 bei 81\%, 2016 bei 89\%, 2017 bei $92 \%, 2018$ bei $90 \%$ und zuletzt 2019 bei 89\% (Daten aus BMI Asylstatistik 2014, 2015, 2016, 2017, 2018, 2019) (vgl. Tabelle 5).

Zudem wird Afghan/inn/en deutlich häufiger nur ein subsidiärer Schutzstatus zugesprochen. Beispielsweise stellten 2017 bei jenen etwa 5.499 Asylwerber/inne/n, die lediglich subsidiären Status erhielten, Afghan/inn/en mit 59\% die stärkste Gruppe. ${ }^{84}$

84 Vgl. Der Standard 2017. 
Tab. 5: Entwicklung der rechtskräftigen Asylentscheidungen Afghanistan, Syrien und Irak (2014-2019)

\begin{tabular}{|c|c|c|c|c|c|c|c|c|}
\hline & \multicolumn{2}{|c|}{$\begin{array}{c}\text { rk. } \\
\text { Asylgewährung }\end{array}$} & \multicolumn{2}{|c|}{$\begin{array}{l}\text { rk. neg. } \\
\text { Asylentscheidung }\end{array}$} & \multicolumn{2}{|c|}{$\begin{array}{c}\text { Sonstige } \\
\text { Entscheidung }\end{array}$} & \multirow[t]{2}{*}{ Gesamt } & \multirow[t]{2}{*}{$\begin{array}{l}\text { rk. Subsidiäre } \\
\text { Schutzgewähr. }\end{array}$} \\
\hline & absolut & in $\%$ & absolut & in $\%$ & absolut & in \% & & \\
\hline \multicolumn{9}{|l|}{2014} \\
\hline Afghanistan & 2.450 & 52,9 & 2.010 & 43,4 & 173 & 3,7 & 4.633 & 1.339 \\
\hline Syrien & 3.604 & 88,2 & 448 & 11,0 & 34 & 0,8 & 4.086 & 324 \\
\hline Irak & 221 & 33,1 & 402 & 63,1 & 24 & 3,8 & 637 & 217 \\
\hline \multicolumn{9}{|l|}{2015} \\
\hline Afghanistan & 2.083 & 30,3 & 2.478 & 36,1 & 2.306 & 33,6 & 6.867 & 1.263 \\
\hline Syrien & 8.114 & 80,5 & 752 & 7,5 & 1.213 & 12,0 & 10.079 & 183 \\
\hline Irak & 637 & 24,5 & 571 & 21,9 & 1.394 & 53,6 & 2.602 & 266 \\
\hline \multicolumn{9}{|l|}{2016} \\
\hline Afghanistan & 1.756 & 25,3 & 2.701 & 38,9 & 2.489 & 35,8 & 6.946 & 1.693 \\
\hline Syrien & 15.528 & 89,2 & 755 & 4,3 & 1.130 & 6,5 & 17.413 & 585 \\
\hline Irak & 1.328 & 29,2 & 1.022 & 22,5 & 2.191 & 48,2 & 4.541 & 633 \\
\hline \multicolumn{9}{|l|}{2017} \\
\hline Afghanistan & 4.274 & 46,7 & 2.957 & 32,3 & 1.925 & 21,0 & 9.156 & 3.248 \\
\hline Syrien & 11.827 & 92,2 & 469 & 3,7 & 528 & 4,1 & 12.824 & 1.194 \\
\hline Irak & 1.160 & 31,9 & 1.481 & 40,7 & 996 & 27,4 & 3.637 & 1.057 \\
\hline \multirow{2}{*}{\multicolumn{9}{|c|}{2018}} \\
\hline & & & & & & & & \\
\hline Afghanistan & 4.979 & 50,4 & 3.986 & 40,3 & 914 & 9,3 & 9.879 & 2.062 \\
\hline Syrien & 4.951 & 89,6 & 472 & 8,5 & 102 & 1,8 & 5.525 & 414 \\
\hline Irak & 731 & 27,5 & 1.314 & 49,5 & 609 & 22,9 & 2.654 & 536 \\
\hline \multirow{2}{*}{\multicolumn{9}{|c|}{2019}} \\
\hline & & & & & & & & \\
\hline Afghanistan & 4.070 & 46,1 & 3.601 & 40,7 & 1.167 & 13,2 & 8.838 & 1.225 \\
\hline Syrien & 2.534 & 88,7 & 235 & 8,2 & 87 & 3,1 & 2.856 & 295 \\
\hline Irak & 300 & 17,1 & 1.121 & 64,0 & 332 & 18,9 & 1.753 & 264 \\
\hline
\end{tabular}

Quellen: BM.I Asylstatistik 2014, 2015, 2016, 2017, 2018 und 2019.

Ein besonderes Problem stellen auch die langen Verfahrensdauern und die zahlreichen offenen Verfahren dar. ${ }^{85}$ Afghanische Asylwerber/innen warten oft deutlich länger als Personen aus anderen Gruppen (z.B. Syrer/innen).

85 Vgl. Der Standard 2017. 
Tab. 6: Offene Verfahren ,internationaler Schutz": Afghanistan, Syrien und Irak im Vergleich (2014-2019)

\begin{tabular}{|c|c|c|c|c|}
\hline & I. Instanz & II. Instanz & \multicolumn{2}{|c|}{ Summe } \\
\hline & & \multicolumn{3}{|c|}{2014} \\
\hline Afghanistan & 4.605 & 2.359 & & 6.964 \\
\hline Syrien & 4.437 & 435 & & 4.872 \\
\hline Irak & 996 & 176 & & 1.172 \\
\hline \multicolumn{5}{|l|}{2015} \\
\hline Afghanistan & 22.811 & 1.456 & & 24.267 \\
\hline Syrien & 18.340 & 329 & & 18.669 \\
\hline Irak & 11.399 & 343 & & 11.742 \\
\hline \multicolumn{5}{|l|}{2016} \\
\hline Afghanistan & 25.086 & 4.697 & & 29.783 \\
\hline Syrien & 8.827 & 985 & & 9.812 \\
\hline \multirow[t]{2}{*}{ Irak } & 8.948 & 1.263 & & 10.211 \\
\hline & $\begin{array}{c}\text { BFA (ohne } \\
\text { Rechtsmittelfrist) }\end{array}$ & Rechtsmittelfrist & Gerichte & Summe \\
\hline \multicolumn{5}{|l|}{2017} \\
\hline Afghanistan & 12.136 & 955 & 11.142 & 24.233 \\
\hline Syrien & 2.446 & 90 & 1.401 & 3.937 \\
\hline Irak & 4.153 & 175 & 3.477 & 7.805 \\
\hline \multicolumn{5}{|l|}{2018} \\
\hline Afghanistan & 1.465 & 139 & 14.489 & 16.093 \\
\hline Syrien & 687 & 40 & 811 & 1.538 \\
\hline Irak & 569 & 58 & 5.142 & 5.769 \\
\hline \multicolumn{5}{|l|}{2019} \\
\hline Afghanistan & 743 & 41 & 9.542 & 10.326 \\
\hline Syrien & 698 & 15 & 591 & 1.304 \\
\hline Irak & 232 & 20 & 4.481 & 4.733 \\
\hline
\end{tabular}

Quellen: Zusammengestellt nach BM.I Asylstatistik 2014, 2015, 2016, 2017, 2018 und 2019. Ab dem Jahr 2017 ändert sich die Gliederung laut BM.I Asylstatistik.

Die lange Verfahrensdauer und die damit verbundene Unsicherheit stellt auch ein großes Hindernis für die Integration von Flüchtlingen dar, zumal es ihnen während der Verfahrensdauer ja nur in Ausnahmefällen (meist nur mit zivilgesellschaftlicher Unterstützung) möglich ist, an Sprach- und anderen Qualifizierungsmaßnahmen teilzunehmen, die jedoch für einen Einstieg in den österreichischen Arbeitsmarkt und eine Teilnahme an der hiesigen Gesellschaft unerlässlich sind. Damit geht nicht nur wertvolle Zeit verloren, sondern das „In-der-Luft-Hängen“" hat auch, wie zahlreiche Studien belegen, einen demotivierenden Effekt. 
Als zusätzlich problematisch erweist sich die seit einigen Monaten forcierte Praxis, afghanische Staatsbürger/innen in die Heimat rückzuführen. Dies hat mittlerweile zu einer starken Verunsicherung unter jenen Afghan/inn/en geführt, die nur ein beschränktes Aufenthaltsrecht in Österreich haben. Darunter fallen z.B. Personen, die zwar einen negativen Asylbescheid erhielten, aber bisher aus humanitären Gründen in Österreich bleiben durften, oder UMF, die bis zu ihrer Volljährigkeit ein Bleiberecht genießen. Mit Erlangen der Volljährigkeit wird nun über ihren Verbleib in Österreich entschieden. Trotz ausgezeichneter Integration in die österreichische Gesellschaft (Schulabschluss, Beginn einer Lehre oder Abschluss derselben) erhalten viele dieser Jugendlichen einen negativen Asylbescheid und sollen nach Afghanistan zurückkehren. Für zahlreiche Afghan/inn/en stellt die Aufforderung zur Rückkehr allerdings ein schwieriges Unterfangen dar, und dies nicht nur wegen der problematischen sicherheitspolitischen und ökonomischen Lage des Landes. Viele Afghan/inn/en haben vor ihrer Flucht nach Österreich jahrelang im Iran oder in Pakistan gelebt oder wurden sogar dort geboren (v.a. viele UMF) und verfügen in „ihrer Heimat“ Afghanistan über keinerlei soziale Netzwerke, die einen Neustart dort ermöglichen könnten.

\subsection{Strukturen der afghanischen Community in Österreich im Spiegel der Statistik}

\subsubsection{Asylstatistik}

Im Falle Afghanistans war bereits das Jahr 2001 durch einen ersten Spitzenwert von nahezu 13.000 Anträgen gekennzeichnet. Dieser Wert halbierte sich im Folgejahr 2002 und ging sodann von 2004 bis 2007 auf Absolutzahlen von weniger als 1.000 Anträgen jährlich zurück. Für 2008 war ein Anstieg auf fast $1.400 \mathrm{zu}$ beobachten, darauf folgte 2009 eine weitere Progression. Die Jahre 2010 und 2013 waren durch erhebliche Rückgänge geprägt, während 2011 eine Verdoppelung der Antragszahlen gegenüber dem Vorjahr erfolgte. 2012 wurde mit mehr als 4.000 Anträgen ein erster hoher Wert erreicht, der sich 2014 steigerte und 2015 mit mehr als 25.000 Anträgen die Höchstzahl erreichte. ${ }^{86}$ Bedingt durch die politischen Entwicklungen auf der EU- und nationalen Ebenen (u.a. Schließung der Westbalkanroute, konsequente Einhaltung der Dublin-Verordnung etc.) gingen die Asylanträge afghanischer Staatsbürger/innen bereits 2016 auf weniger als die Hälfte des Vorjahres zurück und sanken in den Folgejahren weiter ab. Erst 2019 erfolgte ein neuerlicher Anstieg auf 2.853 Anträge (vgl. BMI Asylstatistik, diverse Jahre).

\subsubsection{Statistik des Bevölkerungsstandes}

Die amtliche Statistik spiegelt Differenzen zwischen den Zahlen nach Geburtsland und Staatsbürgerschaft wider. Im Falle Afghanistans sind letztgenannte fast durchwegs

${ }_{86}$ Hinsichtlich der analogen Veränderungen der Antragszahlen und deren Kausalfaktoren bezogen auf Deutschland vgl. RutTig (2017b). 
höher als jene auf Basis des Geburtslandes. Die Ursache dafür liegt in den Rahmenbedingungen der Flucht. Beträchtliche Anteile geflüchteter afghanischer Staatsbürger/innen wurden im Iran oder in Pakistan geboren, was die jahrzehntelange Dauer des Konflikts in dieser Region widerspiegelt (AdelKhaH und Olszewska 2007; Government of PAKISTAN und UNHCR 2007). Da in beiden Staaten Einbürgerungen von Afghan/inn/en kaum erfolgen und diese rezent zunehmend in ihr ursprüngliches Herkunftsland zurückgeschickt werden, behielten diese ihre Staatszugehörigkeit (vgl. dazu BJELICA 2017; Human Rights Watch 2017; IOM 2017).

\section{Tab. 7: Bevölkerung zu Jahresbeginn (01.01.) 2002-2020 nach Staatsangehörig-} keit Afghanistan

\begin{tabular}{|c|c|c|c|}
\hline Jahr & $\begin{array}{c}\text { Afghanistan } \\
\text { absolut }\end{array}$ & Jahr & $\begin{array}{c}\text { Afghanistan } \\
\text { absolut }\end{array}$ \\
\hline 2002 & 2.065 & 2012 & 9.353 \\
\hline 2003 & 2.692 & 2013 & 12.380 \\
\hline 2004 & 3.086 & 2014 & 14.016 \\
\hline 2005 & 3.306 & 2015 & 16.779 \\
\hline 2006 & 3.093 & 2016 & 35.618 \\
\hline 2007 & 3.139 & 2017 & 45.284 \\
\hline 2008 & 3.957 & 2018 & 45.724 \\
\hline 2009 & 4.484 & 2019 & 44.420 \\
\hline 2010 & 5.662 & 2020 & 43.585 \\
\hline 2011 & 6.688 & & \\
\hline
\end{tabular}

Quelle: Statistik Austria, Statistik des Bevölkerungsstandes.

Aussagekräftig sind in der Widerspiegelung der quantitativen Fluchtszenarien vor allem die Veränderungen gegenüber dem jeweils vorangegangenen Jahr, aber auch die längerfristigen Vergleiche innerhalb des gesamten Zeitraums. Die Bevölkerung mit afghanischer Staatsbürgerschaft verzeichnete von 2002 bis 2018 kontinuierliche Zunahmen, die jedoch bis 2008 quantitativ auf ein Plus von wenigen hundert Personen jährlich beschränkt waren. Erst ab 2009 setzten stärkere jährliche Anstiege ein. Von 2012 auf 2013 wurde die Zehntausenderschwelle überschritten. Von 2015 auf 2016 erfolgte ein Wachstumsschub der in Österreich aufhältigen Bevölkerung mit afghanischen Pässen von nahezu 17.000 auf 35.000, dem sich im Analysejahr 2018 ein weiterer Anstieg auf 45.724 Personen anschloss. ${ }^{87}$ Die Jahre 2019 und 2020 waren sodann durch schwächere Rückgänge gekennzeichnet (vgl. Tab. 7).

Die Tabelle 8 ermöglicht eine Analyse der demographischen Struktur der Bevölkerung aus Afghanistan und dokumentiert, dass in summa die afghanische Community im Jahr 2019 43.073 Personen umfasste. Die Verteilung nach Alter und Geschlecht

${ }_{87}$ Neuerlich ist als wesentlicher Kausalfaktor auf die Veränderung der Sicherheitslage im Herkunftsland hinzuweisen (vgl. Clark 2017; Sigar 2017; UnAma 2017a). 
dokumentiert den mit 68\% überproportional hohen Männeranteil unter der in Afghanistan geborenen Wohnbevölkerung in Österreich. Die Analyse nach dem Kriterium der Altersstruktur belegt, dass in summa eine sehr junge Zuwanderung vorliegt, aber auch die Kohorten mittleren Alters sind stark repräsentiert, denn Personen von 25 bis 49 Jahren stellen mit 43,6\% die dominierende Altersgruppe dar. Mit 2,6\% ist der Kleinkinderanteil bei den in Afghanistan Geborenen gering, Kinder von 6 bis 14 Jahren finden sich in dieser Herkunftsgruppe mit einem Anteil von 12,1\%. ${ }^{88}$ Für die Jugendlichen im Alter zwischen 15 und 24 Jahren ist deren besonders hohe Präsenz $(34,7 \%)$ hervorzuheben. Diese spiegelt eine spezifische Migrationsstrategie wider, im Rahmen derer sich männliche Jugendliche eigeninitiativ auf den Weg nach Europa machen oder von den Herkunftsfamilien finanziell unterstützt, gezielt ausgesandt werden (vgl. ECHAVEZ et al. 2014; KAZEMi 2016). Am seltensten finden sich ältere Menschen, denn lediglich knapp 7\% haben das 50. Lebensjahr bereits überschritten.

Tab. 8: Bevölkerung nach Geburtsland Afghanistan, Alter und Geschlecht, 01-01-2019

\begin{tabular}{|l|r|r|}
\hline \multicolumn{1}{|c|}{ Geschlecht } & absolut & \multicolumn{1}{c|}{ in $\%$} \\
\hline Männer & 29.303 & 68,0 \\
\hline Frauen & 13.770 & 32,0 \\
\hline insgesamt & $\mathbf{4 3 . 0 7 3}$ & $\mathbf{1 0 0 , 0}$ \\
\hline Altersgruppen & absolut & in $\%$ \\
\hline 0 bis 5 Jahre & 1.139 & 2,64 \\
\hline 6 bis 14 Jahre & 5.213 & 12,10 \\
\hline 15 bis 17 Jahre & 2.695 & 6,26 \\
\hline 18 bis 24 Jahre & 12.245 & 28,42 \\
\hline 25 bis 34 Jahre & 12.017 & 27,90 \\
\hline 35 bis 49 Jahre & 6.740 & 15,65 \\
\hline 50 bis 64 Jahre & 2.357 & 5,47 \\
\hline 65 Jahre und älter & 667 & 1,55 \\
\hline insgesamt & $\mathbf{4 3 . 0 7 3}$ & $\mathbf{1 0 0 , 0}$ \\
\hline
\end{tabular}

Quellen: Statistik Austria, Statistik des Bevölkerungsstandes; eigene Berechnung.

Tabelle 9 spiegelt die geringe Präsenz der Afghan/inn/en an der Wohnbevölkerung in Österreich insgesamt sowohl nach dem Kriterium des Geburtslandes als auch nach jenem der Staatsbürgerschaft wider. Der jeweilige Anteil betrug 2019 nur rund 0,5\%. Etwas höher, im Vergleich zu anderen Herkunftsgruppen allerdings auch bescheiden, fällt der Anteil an allen im Ausland Geborenen aus, der im Falle Afghanistans knapp 2,5\% beträgt. Am höchsten ist mit etwas über 3\% der Anteilswert unter den ausländischen Staatsangehörigen.

88 An Analysen zur Migration von Kindern aus Afghanistan vgl. SERrano und Voon (2013) sowie UNHCR (2010). 
Tab. 9: Anteile von Afghan/inn/en an der Bevölkerung insgesamt und an allen im Ausland Geborenen bzw. an allen ausländischen Staatsangehörigen, 2019

\begin{tabular}{|l|r|}
\hline Geburtsland & \\
\hline Anteil an der Wohnbevölkerung & 0,49 \\
\hline Anteil an allen im Ausland Geborenen & 2,49 \\
\hline Staatsangehörigkeit & \\
\hline Anteil an der Wohnbevölkerung & 0,50 \\
\hline Anteil an allen ausländischen Staatsangehörigen & 3,08 \\
\hline
\end{tabular}

Quellen: Statistik Austria, Statistik des Bevölkerungsstandes; eigene Berechnung.

Tab. 10: Einbürgerungszahlen afghanischer Staatsbürger/innen 1981-2019

\begin{tabular}{|c|r|r|}
\hline $\begin{array}{c}\text { Einbürgerungs- } \\
\text { jahr }\end{array}$ & abs. & in \% \\
\hline 1981 & 19 & 0,4 \\
\hline 1982 & 4 & 0,1 \\
\hline 1983 & 7 & 0,2 \\
\hline 1984 & 6 & 0,1 \\
\hline 1985 & 17 & 0,4 \\
\hline 1986 & 5 & 0,1 \\
\hline 1987 & 13 & 0,3 \\
\hline 1988 & 34 & 0,7 \\
\hline 1989 & 43 & 0,9 \\
\hline 1990 & 31 & 0,8 \\
\hline 1991 & 44 & 0,9 \\
\hline 1992 & 46 & 1,0 \\
\hline 1993 & 20 & 0,4 \\
\hline 1994 & 21 & 0,5 \\
\hline 1995 & 30 & 0,6 \\
\hline 1996 & 16 & 0,3 \\
\hline 1997 & 31 & 0,7 \\
\hline 1998 & 41 & 0,9 \\
\hline 1999 & 56 & 1,2 \\
\hline 2000 & 70 & 1,5 \\
\hline
\end{tabular}

\begin{tabular}{|c|r|r|}
\hline $\begin{array}{c}\text { Einbürgerungs- } \\
\text { jahr }\end{array}$ & abs. & in \% \\
\hline 2001 & 44 & 0,9 \\
\hline 2002 & 69 & 1,5 \\
\hline 2003 & 135 & 2,9 \\
\hline 2004 & 322 & 7,0 \\
\hline 2005 & 454 & 9,8 \\
\hline 2006 & 261 & 5,6 \\
\hline 2007 & 43 & 0,9 \\
\hline 2008 & 106 & 2,3 \\
\hline 2009 & 108 & 2,3 \\
\hline 2010 & 113 & 2,4 \\
\hline 2011 & 157 & 3,4 \\
\hline 2012 & 179 & 3,9 \\
\hline 2013 & 208 & 4,5 \\
\hline 2014 & 232 & 5,0 \\
\hline 2015 & 187 & 4,0 \\
\hline 2016 & 332 & 7,2 \\
\hline 2017 & 424 & 9,2 \\
\hline 2018 & 328 & 7,0 \\
\hline 2019 & 372 & 8,0 \\
\hline Insgesamt & $\mathbf{4 . 6 2 8}$ & $\mathbf{1 0 0 , 0}$ \\
\hline & & \\
\hline
\end{tabular}

Quelle: Statistik Austria.

Die Einbürgerung repräsentiert einen wichtigen formalen Akt im Rahmen der Integrationsbiographie. Die Tabelle 10 beinhaltet eine Zeitreihe der Entwicklung der Einbürgerungszahlen über einen Zeitraum von nahezu 40 Jahren und spiegelt eine Reihe von Veränderungen im Zeitverlauf wider. Die Einbürgerungszahlen von Immigrant/inn/ en aus Afghanistan bewegten sich die 1980er Jahre hindurch auf einem sehr niedrigen Niveau, da diese Community zahlenmäßig in Österreich nur schwach repräsentiert war. 
Erst gegen Ende der 1990er Jahre stiegen die Zahlen auf Werte zwischen 40 und 50 Personen an. Allerdings waren damals die Hürden für die Einbürgerung im Vergleich zur rezenten Rechtslage noch vergleichsweise niedrig. Aufgrund der geringen Absolutzahlen bewegten sich auch die Anteilswerte auf einem niedrigen Niveau. Erst nach der Jahrtausendwende verzeichneten die Einbürgerungen einen zunächst geringen und im Zeitraum von 2003 bis 2005 einen deutlichen Anstieg, wobei ein Spitzenwert von 454 im Jahre 2005 erzielt wurde. Diese Absolutzahl entsprach einem Anteil von 13\% an allen Einbürgerungen von afghanischen Staatsbürger/inne/n im Analysezeitraum. Nach einer signifikanten Reduktion 2007 stiegen die Zahlen in den Folgejahren ab 2008 auf Werte von über 100 jährlich an. Der Wert von 200 wurde in den Jahren 2013 und 2014 jeweils überschritten. Mit 332 Einbürgerungen afghanischer Bürger/innen 2016 wurde der zweithöchste Zahlenwert im Verlauf des gesamten Analysezeitraumes erreicht. Diese Zahl entsprach einem Anteil von 9,5\% aller Einbürgerungen von Personen aus Afghanistan im gesamten Betrachtungszeitraum. Während 2017 der zweithöchste Wert seit 2005 erreicht wurde, sanken die Zahlen danach wieder ab.

Tabelle 11 zeigt die Männerdominanz der Zuwanderung nach dem Geburtsland Afghanistan in allen Bundesländern sowie die zum Teil beträchtlichen Unterschiede in den zahlen- und anteilsmäßigen Geschlechterrelationen zwischen den Bundesländern. Die komparative Analyse ergibt, dass die Frauenanteile vor allem in Oberösterreich, aber auch in Wien am höchsten sind. Eine Erklärungshypothese könnte im stärker urban geprägten Charakter dieser beiden Bundesländer mit den großstädtischen Agglomerationen des Wiener und Linzer Zentralraumes liegen. In welchem Ausmaß hier die vielfältigeren Arbeitsmöglichkeiten für Frauen eine Rolle spielen, kann in Ermangelung von entsprechenden Untersuchungen nur gemutmaßt werden. Frauen finden oftmals im informellen Sektor, wie etwa in persönlichen Dienstleistungen, in Reinigung, Pflege und Service, also im geringer qualifizierten Segment des Arbeitsmarktes, leichter eine Beschäftigung als Männer. ${ }^{89}$ Inwieweit dies für Afghaninnen gelten könnte, wissen wir nicht. Der ländliche Raum offeriert im Vergleich dazu nur wenig Beschäftigungsperspektiven, sieht man von der Branche Fremdenverkehr und Gastronomie ab, und vor allem auch einen schwierigen Zugang zu Weiterbildungsangeboten und Kinderbetreuungsinfrastruktur. Gegen diese Hypothese spricht aber im Fall der afghanischen Frauen, dass Frauen kulturbedingt und durch spezifische Fluchtmuster in dieser stark traditionell geprägten Herkunftsgruppe ihren Männern in der Regel nach Österreich nachziehen. Daher sind eigenständige Wohnstandortentscheidungen zumindest in der Frühphase des Aufenthalts in Österreich bei einem Gros der afghanischen Frauen nicht anzunehmen.

Die niedrigsten Frauenanteile sind in Salzburg, Vorarlberg und im Burgenland festzustellen, bei jeweils über einem Drittel liegt die Frauenpräsenz in Kärnten, Niederösterreich und Tirol.

89 Eine umfassende Analyse der Arbeitsmarktintegration von Migrantinnen, z.B. im informellen Sektor, beinhaltet die Studie von KonTos (2011). 
Tab. 11: Bevölkerung nach Geburtsland Afghanistan, Geschlecht und Bundesland, $2017^{90}$

\begin{tabular}{|l|r|r|}
\hline Bundesland & absolut & \multicolumn{1}{c|}{ in \% } \\
\hline Burgenland & 931 & 100,0 \\
\hline Männer & 675 & 72,5 \\
\hline Frauen & 256 & 27,5 \\
\hline Kärnten & 2.101 & 100,0 \\
\hline Männer & 1.393 & 66,3 \\
\hline Frauen & 708 & 33,7 \\
\hline Niederösterreich & 5.072 & 100,0 \\
\hline Männer & 3.431 & 67,6 \\
\hline Frauen & 1.641 & 32,4 \\
\hline Oberösterreich & 6.991 & 100,0 \\
\hline Männer & 4.391 & 62,8 \\
\hline Frauen & 2.600 & 37,2 \\
\hline Salzburg & 2.372 & 100,0 \\
\hline Männer & 1.817 & 76,6 \\
\hline Frauen & 555 & 23,4 \\
\hline Steiermark & 5.245 & 100,0 \\
\hline Männer & 3.498 & 66,7 \\
\hline Frauen & 1.747 & 33,3 \\
\hline Tirol & 2.787 & 100,0 \\
\hline Männer & 1.876 & 67,3 \\
\hline Frauen & 911 & 32,7 \\
\hline Vorarlberg & 1.507 & 100,0 \\
\hline Männer & 1.064 & 70,6 \\
\hline Frauen & 443 & 29,4 \\
\hline Wien & 17.066 & 100,0 \\
\hline Männer & 11.089 & 65,0 \\
\hline Frauen & 5.977 & 35,0 \\
\hline Insgesamt & 25.673 & $\mathbf{1 0 0 , 0}$ \\
\hline Männer & 10.934 & 58,3 \\
\hline Frauen & & 41,7 \\
\hline
\end{tabular}

Quellen: Statistik Austria, Abgestimmte Erwerbsstatistik, eigene Berechnung.

\subsubsection{Räumliche Verteilung und soziale Struktur der afghanischen Community}

Tabelle 12 weist Wien als jenes Bundesland mit der zahlenmäßig größten afghanischen Community aus. Mit erheblichem Abstand folgen dann die flächenmäßig großen Bundesländer Oberösterreich, Niederösterreich und die Steiermark. Auch in diesen 90 Die Daten für 2017 waren zum Zeitpunkt der Erstellung dieses Berichts die rezentesten verfügbaren (vgl. <https://www.statistik.at/web_de/statistiken/menschen_und_gesellschaft/ bevoelkerung/volkszaehlungen_registerzaehlungen_abgestimmte_erwerbsstatistik/bevoelkerung_nach_demographischen_merkmalen/index.html $>$ ). 
Bundesländern ist die afghanische Community vor allem auf die größeren urbanen Zentren konzentriert.

Tab. 12: Bevölkerung aus Afghanistan (Geburtsland) nach Bundesländern, 2019

\begin{tabular}{|l|r|r|}
\hline \multicolumn{1}{|c|}{ Bundesland } & \multicolumn{1}{c|}{ absolut } & \multicolumn{1}{c|}{ in \% } \\
\hline Burgenland & 643 & 1,49 \\
\hline Kärnten & 1.666 & 3,87 \\
\hline Niederösterreich & 4.268 & 9,90 \\
\hline Oberösterreich & 6.705 & 15,57 \\
\hline Salzburg & 2.329 & 5,41 \\
\hline Steiermark & 4.769 & 11,07 \\
\hline Tirol & 2.773 & 6,44 \\
\hline Vorarlberg & 1.537 & 3,57 \\
\hline Wien & 18.383 & 42,68 \\
\hline insgesamt & $\mathbf{4 3 . 0 7 3}$ & $\mathbf{1 0 0 , 0 0}$ \\
\hline
\end{tabular}

Quellen: Statistik Austria, Statistik des Bevölkerungsstandes; eigene Berechnung.

Die Tabelle 13 dokumentiert die dominierende Rolle der Bundeshauptstadt als der wohnungs- und arbeitsmarktbedingte Zuwanderungspol für die Fluchtmigration aus Afghanistan. Während sich die Community bis 2009 noch auf weniger als 2.000 Personen belief, war sie dennoch die zahlenmäßig größte unter allen österreichischen Bundesländern. Ein stärkerer jährlicher Wachstumstrend war ab 2010 zu verzeichnen. 2015/16 schnellte die Zahl um mehr als 65\% nach oben, aber bereits ab 2017 gingen die Zuwachsraten wieder deutlich zurück. Die Männerdominanz der afghanischen Community in Wien kam 2018 darin zum Ausdruck, dass 11.301 Männern mit afghanischer Staatsbürgerschaft nur 5.722 Frauen gegenüberstanden. Dies entsprach einem

Tab. 13: Bevölkerung aus Afghanistan (nach Staatsangehörigkeit) in Wien, 2006-2019

\begin{tabular}{|r|r|r|}
\hline Jahr & absolut & Veränderung \\
\hline 2006 & 1.175 & -- \\
\hline 2007 & 1.165 & $-9,9$ \\
\hline 2008 & 1.694 & $+45,4$ \\
\hline 2009 & 1.738 & $+2,6$ \\
\hline 2010 & 2.350 & $+35,2$ \\
\hline 2011 & 2.902 & $+23,5$ \\
\hline 2012 & 3.873 & $+33,5$ \\
\hline
\end{tabular}

\begin{tabular}{|r|r|r|}
\hline Jahr & absolut & Veränderung \\
\hline 2013 & 4.743 & $+22,5$ \\
\hline 2014 & 5.826 & $+22,8$ \\
\hline 2015 & 7.558 & $+29,7$ \\
\hline 2016 & 12.479 & $+65,1$ \\
\hline 2017 & 14.582 & $+16,9$ \\
\hline 2018 & 17.023 & $+16,7$ \\
\hline 2019 & 17.767 & $+4,4$ \\
\hline
\end{tabular}

Quellen: Statistik Austria, Statistik des Bevölkerungsstandes; eigene Berechnung. Veränderung: gegenüber dem Vorjahr. 
Anteil letzterer von nur 33,6\%, der sodann 2019 geringfügig auf 35\% (6.329 Frauen gegenüber 11.548 Männern) anwuchs. ${ }^{91}$

Die Karte 1 verdeutlicht für 2016, in welch geringen Anteilen die in Afghanistan geborene Wohnbevölkerung im Vergleich zu anderen Immigrant/inn/engruppen (wie etwa den ehemaligen „Gastarbeiter/inne/n“ aus der Türkei und Exjugoslawien) in der österreichischen Wohnbevölkerung präsent war. Die höchsten Anteilswerte im Ausmaß zwischen 0,53 und $0,85 \%$, aber auch jene zwischen 0,35 und $0,53 \%$ waren in erster Linie in Wien und in politischen Bezirken der flächenmäßig großen Bundesländer Niederund Oberösterreich sowie Steiermark präsent. Diese Verteilung hat mit der Attraktivität der urban geprägten Teile Österreichs und deren Arbeitsmärkten zu tun. So treten die Agglomeration Wien, der Großraum Linz, aber auch die Landeshauptstädte St. Pölten, Graz, Innsbruck und Klagenfurt als räumliche Einheiten mit besonders hohen Anteilen von in Afghanistan geborener Bevölkerung hervor. Was die Bezirke im Norden der Steiermark sowie im nördlichen Oberösterreich, des Weiteren im südlichen Burgenland

\section{Karte 1: Anteil von in Afghanistan geborener Bevölkerung an der Wohnbevölke-} rung, 2016

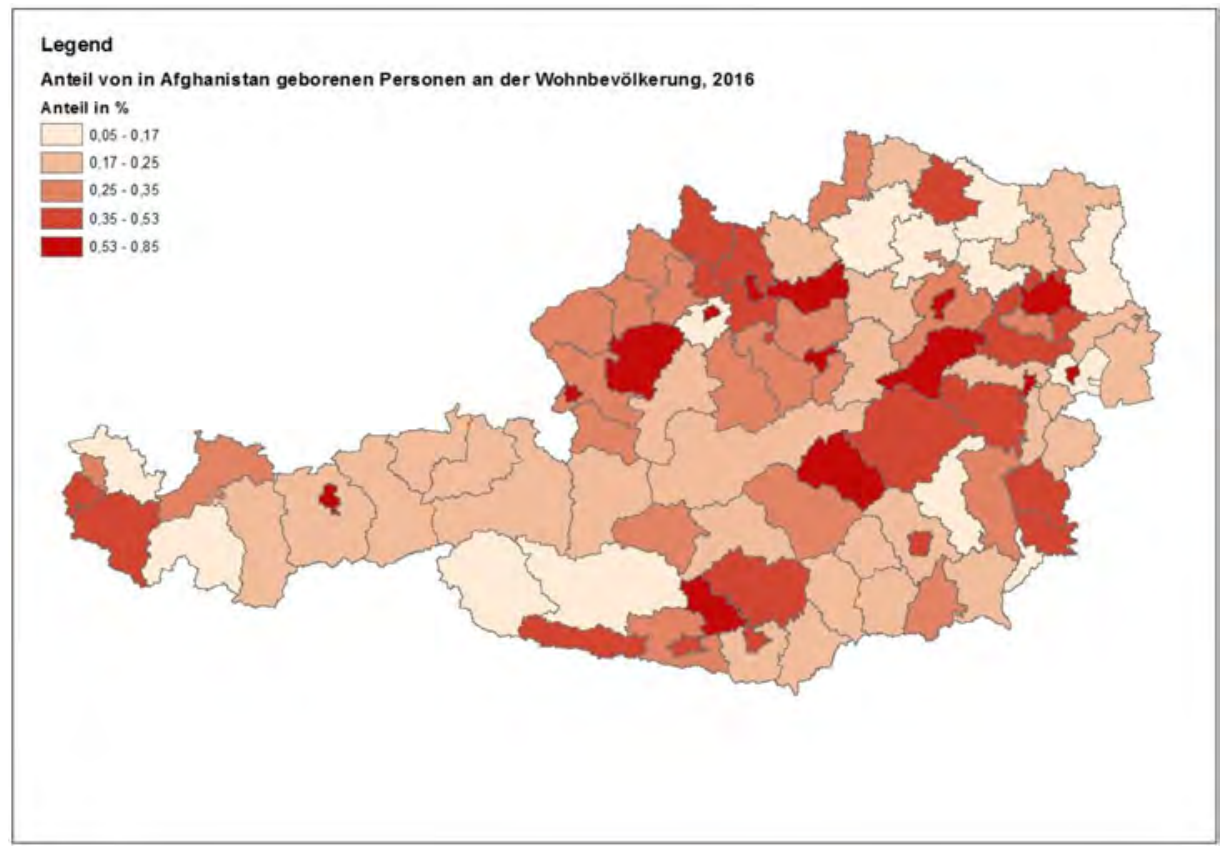

Quelle: Statistik Austria.

${ }_{91}$ Vgl. <https://www.wien.gv.at/statistik/bevoelkerung/tabellen/bevoelkerung-staat-geschl-zr. html>. 
und in Kärnten anbelangt, in denen die Prozentanteile relativ hoch ausfielen, so lässt sich als weiterer Kausalfaktor die Verteilung der Asylquartiere ausmachen, von denen auch zahlreiche größere in Gemeinden bzw. Bezirken des ländlichen Raumes lokalisiert waren. Damit ist, trotz hoher Binnenmigration in die Städte, ein gewisses Verteilungsmuster vorgegeben, das sich aufgrund der Schließung von Quartieren jedoch in dauernder Veränderung befindet.

Im Folgenden wird zunächst, basierend auf Daten der Statistik Austria (Abgestimmte Erwerbsstatistik), auf den ökonomischen Background, Ausbildungsstand und die Lebensformen der in Österreich residierenden Afghan/inn/en eingegangen. ${ }^{92}$

Tab. 14: Erwerbsstatus nach Staatsangehörigkeit bzw. Geburtsland (Personen, 15 Jahre und älter), 2014

\begin{tabular}{|l|r|r|}
\hline & $\begin{array}{c}\text { Staatsangehörigkeit } \\
\text { Afghanistan }\end{array}$ & $\begin{array}{c}\text { Geburtsland } \\
\text { Afghanistan }\end{array}$ \\
\hline Erwerbspersonen & 5.742 & 7.473 \\
\hline erwerbstätig & 2.941 & 4.237 \\
\hline arbeitslos & 2.801 & 3.236 \\
\hline Arbeitslosenquote (Pers. 15 Jahre und älter) & 48,8 & 43,3 \\
\hline
\end{tabular}

Quelle: STATcube - Statistische Datenbank von STATISTIK AUSTRIA.

Die Tabelle 14 dokumentiert zum einen die bereits im Jahr 2014, also noch vor Einsetzen der so genannten „refugee crisis“, hohen Arbeitslosenquoten, zum anderen spiegelt sie die gerade in Bezug auf die Arbeitslosigkeit deutlichen Unterschiede zwischen den Kategorien „Staatsangehörigkeit“ und „Geburtsland“ wider. In der Kategorie „Geburtsland“ waren die Arbeitslosenquoten im Vergleich geringer, was auf den höheren Anteil an Eingebürgerten und somit bereits länger in Österreich aufhältigen Personen zurückzuführen war. Bei Afghan/inn/en lag diese bei 43,3\%. Die schlechter ausgebildeten Afghan/inn/en wiesen allerdings ein niedrigeres Aspirationsniveau in Bezug auf die angestrebten Berufspositionen auf.

Tabelle 15 spiegelt den Erwerbsstatus und die altersmäßige Zusammensetzung der Geflüchteten nach dem Geburtsland und nach der Staatsangehörigkeit wider. Vor allem die Kategorie Staatsangehörigkeit spiegelt die hohe Anzahl von unter 15-jährigen Personen (3.499) mit afghanischer Staatsbürgerschaft wider. Schüler/innen und Studierende über 15 Jahre sind stark vertreten. Dazu kommen für Afghanistan 6.388 sonstige Nichterwerbspersonen. Die Zahl der Pensionsbezieher/innen ist gering, was auf die rezenten Zuwanderungszeitpunkte und die generell ,junge“ Altersstruktur hinweist. Der

$92 \quad$ Die Abgestimmte Erwerbsstatistik wird seit 2008 jährlich gemäß Anlage II Bundesstatistikgesetz erstellt. Das Konzept der Abgestimmten Erwerbsstatistik wurde im Rahmen der Probezählung 2006 entwickelt und basiert auf Administrativdaten (Stichtag 31.10.). 
Tab. 15: Erwerbsstatus nach Staatsangehörigkeit bzw. Geburtsland (Personen, 15 Jahre und älter), 2014

\begin{tabular}{|l|r|r|}
\hline & \multicolumn{1}{|c|}{$\begin{array}{c}\text { Staatsangehörigkeit } \\
\text { Afghanistan }\end{array}$} & \multicolumn{1}{c|}{$\begin{array}{c}\text { Geburtsland } \\
\text { Afghanistan }\end{array}$} \\
\hline aktiv erwerbstätig & 2.902 & 4.177 \\
\hline $\begin{array}{l}\text { temporär abwesend } \\
\text { (Annahme aufrechtes Dienstverhältnis) }\end{array}$ & 39 & 60 \\
\hline arbeitslos & 2.801 & 3.236 \\
\hline Personen unter 15 Jahren & 3.499 & 2.243 \\
\hline Personen mit Pensionsbezug & 8 & 71 \\
\hline $\begin{array}{l}\text { Schüler/innen, Studierende } \\
\text { 15 Jahre und älter }\end{array}$ & 745 & 1.001 \\
\hline Sonstige Nichterwerbspersonen & 6.388 & 6.765 \\
\hline insgesamt & $\mathbf{1 6 . 3 8 2}$ & $\mathbf{1 7 . 5 5 3}$ \\
\hline
\end{tabular}

Quelle: STATcube - Statistische Datenbank von STATISTIK AUSTRIA.

Anteil der aktiv Erwerbstätigen afghanischer Staatsbürgerschaft liegt bei 17,7\%. Führt man die Analyse auf Basis des Geburtslandes durch, so sind die Anteile der Erwerbstätigen höher: Im Falle Afghanistans liegt dieser Anteil bei 23,8\%. Zugleich stellen, nach dem Geburtsland berechnet, auch die Pensionsbezieher/innen aufgrund ihrer längeren Aufenthaltsdauer eine größere Gruppe dar.

Tab. 16: Erwerbsstatus und -quoten nach Staatsangehörigkeit bzw. Geburtsland (Personen im Alter 15 bis 64 Jahre), 20140

\begin{tabular}{|l|r|r|}
\hline & $\begin{array}{c}\text { Staatsangehörigkeit } \\
\text { Afghanistan }\end{array}$ & $\begin{array}{c}\text { Geburtsland } \\
\text { Afghanistan }\end{array}$ \\
\hline Erwerbspersonen & 5.740 & 7.462 \\
\hline erwerbstätig & 2.940 & 4.229 \\
\hline arbeitslos & 2.800 & 3.233 \\
\hline Nichterwerbspersonen & 6.988 & 7.605 \\
\hline insgesamt & $\mathbf{1 2 . 7 2 8}$ & $\mathbf{1 5 . 0 6 7}$ \\
\hline Erwerbstätigenquote der 15- bis 64-jährigen & 23,1 & 28,1 \\
\hline
\end{tabular}

Quelle: STATcube - Statistische Datenbank von STATISTIK AUSTRIA.

Die Tabelle 16 dokumentiert, dass die Erwerbstätigenquoten bezogen auf die Kategorie Geburtsland über jener nach der Staatsbürgerschaft lagen. Die Relationen zwischen Erwerbspersonen, die sich aus Erwerbstätigen plus den arbeitslos Gemeldeten zusammensetzen, und Nichterwerbspersonen divergierten. In Bezug auf die Staatsangehörigkeit betrug der Anteil der Erwerbspersonen im Falle Afghanistans 45\%, bezogen auf die Variable Geburtsland 49,5\%. 
Die Tabelle 17 belegt, dass Tätigkeiten in Arbeiterberufen dominieren. Bezogen auf die Staatsangehörigkeit nahmen 59,8\% der Erwerbspersonen aus Afghanistan sozialrechtlich Arbeiterpositionen ein, bezogen auf die Variable Geburtsland war der Anteil $(59,1 \%)$ nahezu ident. Dies spiegelt also auch nach längerem Aufenthalt eine Stabilität der Anteile der Arbeiterberufe unter den Immigrant/inn/en aus Afghanistan wider.

Tab. 17: Stellung im Beruf nach Staatsangehörigkeit bzw. Geburtsland, 2014

\begin{tabular}{|l|r|r|}
\hline \multicolumn{1}{|c|}{ Stellung im Beruf } & $\begin{array}{c}\text { Staatsangehörigkeit } \\
\text { Afghanistan }\end{array}$ & $\begin{array}{c}\text { Geburtsland } \\
\text { Afghanistan }\end{array}$ \\
\hline Arbeiter/innen & 3.435 & 4.416 \\
\hline Angestellte & 508 & 1.051 \\
\hline sonstige unselbständige Erwerbstätige & 28 & 70 \\
\hline Arbeitgeber & 45 & 87 \\
\hline Selbständige & 71 & 140 \\
\hline mithelfende Familienangehörige & 16 & 19 \\
\hline $\begin{array}{l}\text { arbeitslos, Stellung im Beruf unbekannt oder } \\
\text { erstmals Arbeit suchend }\end{array}$ & 1.639 & 1.690 \\
\hline Nichterwerbspersonen & 10.640 & 10.080 \\
\hline insgesamt & $\mathbf{1 6 . 3 8 2}$ & $\mathbf{1 7 . 5 5 3}$ \\
\hline
\end{tabular}

Quelle: STATcube - Statistische Datenbank von STATISTIK AUSTRIA.

In einem Gegensatz dazu standen die Ergebnisse des Vergleichs der Angestelltenzahlen. So waren die Angestelltenzahlen bei in Afghanistan Geborenen mehr als doppelt so hoch als bei den Staatsangehörigen dieses zentralasiatischen Landes. Dies dokumentiert das mit längerer Aufenthaltsdauer und der Einbürgerung einhergehende Avancement der Immigrant/inn/en, denen es gelingt, in vermehrtem Maß in Angestelltenberufen tätig zu sein. Da Lehrlinge unter den Angestellten inkludiert sind, kann allerdings nicht spezifiziert werden, in welchem Ausmaß für diesen Anstieg auch steigende Lehrlingszahlen verantwortlich zeichnen.

Gering war der Anteil der Entrepreneurs. Bezogen auf die Staatsangehörigkeit lag er bei 1,4\%, bezogen auf alle Erwerbspersonen nach Geburtsland bei 1,9\%. Gering war auch die Präsenz mithelfender Familienangehöriger, eine Kategorie, die im migrantischen Unternehmersegment mit seiner ausgeprägten Involvierung von Familienmitgliedern ansonsten stark repräsentiert ist (vgl. KOHLBACHER und REEGER 2013).

Dass es sich bei einem Großteil der afghanischen Unternehmen um EPU handelte wird durch die folgende Relation belegt: So standen bei der Analyse nach dem Geburtsland 140 Selbständige aus Afghanistan 87 Arbeitgeber/inne/n gegenüber. 
Tab. 18: Laufende Ausbildung nach Staatsangehörigkeit bzw. Geburtsland, 2014

\begin{tabular}{|l|r|r|}
\hline \multicolumn{1}{|c|}{ Laufende Ausbildung in: } & $\begin{array}{c}\text { Staatsangehörigkeit } \\
\text { Afghanistan }\end{array}$ & $\begin{array}{c}\text { Geburtsland } \\
\text { Afghanistan }\end{array}$ \\
\hline Volksschule & 868 & 691 \\
\hline Hauptschule/Neue Mittelschule & 743 & 769 \\
\hline Sonderschule & 65 & 64 \\
\hline Polytechnische Schule & 125 & 131 \\
\hline AHS Unterstufe & 105 & 130 \\
\hline AHS Oberstufe & 261 & 398 \\
\hline Berufsschule/Lehre & 360 & 399 \\
\hline Berufsbildende mittlere Schule & 112 & 158 \\
\hline Berufsbildende höhere Schule & 192 & 271 \\
\hline Gesundheitsschule & 3 & 3 \\
\hline Kolleg & 6 & - \\
\hline Hochschulverwandte Lehranstalt & - & 254 \\
\hline Hochschule & 100 & 36 \\
\hline \multicolumn{1}{|c|}{ Sonstige laufende Bildung } & 37 & 14.027 \\
\hline Keine laufende Bildung & 13.190 & 214 \\
\hline Schulbesuch unbekannt & 251 & \\
\hline
\end{tabular}

Quelle: STATcube - Statistische Datenbank von STATISTIK AUSTRIA.

Tabelle 18 illustriert das Ausmaß der Personen in laufender Ausbildung. Am zahlreichsten war die Kategorie der nicht in einer laufenden Ausbildung befindlichen Personen. Zahlenmäßig dominierte bei den in Ausbildung befindlichen Personen der Besuch von Pflichtschulen, relativ geringe Zahlen entfielen auf den Besuch einer AHS-Unterstufe. Deutlich höher war durchwegs die Partizipation in einer Oberstufe einer AHS, die Zahl war mit 261 bzw. 398 in erster Linie im Segment afghanischer StaatsbürgerInnen bzw. Personen mit Geburtsland Afghanistan anteilsmäßig hoch. Berufsschulen/Lehrausbildungen wurden ebenfalls überdurchschnittlich häufig absolviert ebenso wie berufsbildende mittlere und höhere Schulen. Auch über einige soziologische Basisdaten erteilt die Abgestimmte Erwerbsstatistik 2014 Auskunft (vgl. Tab. 19). Von den 16.382 Personen mit afghanischer Staatsbürgerschaft lebten $77,7 \%$ in Privathaushalten und mit 3.656 Personen (22,3\%) ein verhältnismäßig hoher Anteil in Nichtprivathaushalten. Personen in Partnerschaften ohne Kind (5,7\%) sowie Alleinlebende (6\%) stellten mit jeweils über 900 Personen nur kleine Gruppen dar. Fast 3.700 afghanische Staatsbürger/innen lebten mit ihren Kindern im Haushalt. Von den 4.435 Töchtern bzw. Söhnen befanden sich 62,8\% im Alter unter 15 Jahren und 37,2\% waren zum Erhebungszeitpunkt bereits älter als 15. Sonstige Haushaltsmitglieder waren mit fast 2.700 in verhältnismäßig hoher Zahl zu finden. 
Tab. 19: Lebensform nach Staatsangehörigkeit bzw. Geburtsland, 2014

\begin{tabular}{|c|r|r|}
\hline & $\begin{array}{c}\text { Staatsangehörigkeit } \\
\text { Afghanistan }\end{array}$ & $\begin{array}{c}\text { Geburtsland } \\
\text { Afghanistan }\end{array}$ \\
\hline Personen in Privathaushalten & $\mathbf{1 2 . 7 2 6}$ & $\mathbf{1 4 . 0 4 5}$ \\
\hline Pers. in Partnerschaft lebend ohne Kind & 934 & 1.147 \\
\hline Personen mit Kind(ern) & 3.692 & 4.967 \\
\hline Tochter/Sohn & 4.435 & 3.865 \\
\hline unter 15 Jahren & 2.783 & 1.656 \\
\hline ab 15 Jahren & 1.652 & 2.209 \\
\hline Allein lebend & 975 & 1.173 \\
\hline Sonstige Haushaltsmitglieder & 2.690 & 2.893 \\
\hline Personen in Nicht-Privathaushalten* & $\mathbf{3 . 6 5 6}$ & $\mathbf{3 . 5 0 8}$ \\
\hline Gesamt & $\mathbf{1 6 . 3 8 2}$ & $\mathbf{1 7 . 5 5 3}$ \\
\hline
\end{tabular}

Quelle: STATISTIK AUSTRIA, Abgestimmte Erwerbsstatistik 2014 mit Stichtag 31.10.;

*Personen in Anstalten bzw. Wohnungslose

Erheblich höher war der Anteil der in Privathaushalten lebenden afghanischstämmigen Bevölkerung, nämlich 80\% (14.045 Personen), bei einer Analyse der Community nach dem Kriterium des Geburtslandes. 20\% oder 3.508 Personen, nach dem Geburtsland berechnet, lebten in Nichtprivathaushalten. Alleinlebende sowie Personen in Partnerschaften ohne Kind waren hier ebenfalls in höheren Anteilen zu finden, nämlich zu 6,7\% bzw. zu 6,5\%. Jedenfalls spiegeln diese Werte eine im Vergleich zur österreichischen Gesamtbevölkerung noch geringe Singularisierungstendenz wider. Fast 5.000 Personen, gaben an, mit ihren Kindern im selben Haushalt zu leben, wobei sich von den 3.856 Töchtern bzw. Söhnen 42,8\% im Alter unter 15 und 57,2\% im Alter über 15 Jahren befanden.

\subsection{Ankommen in Österreich}

Die Mehrzahl der heute in Österreich lebenden Afghan/inn/en sind entweder Asylwerber/innen, anerkannte oder inzwischen eingebürgerte Personen. Sie stammen aus unterschiedlichen ethnischen Gruppierungen, gehören verschiedenen sozialen Schichten an und haben einen sehr divergierenden Bildungshintergrund, von afghanischen Spitzenbeamten bis hin zu einer großen Zahl von Analphabet/inn/en. ${ }^{93}$

Insgesamt hat die afghanische Community seit den späten 1960er und frühen 1970er Jahre, als die ersten Afghan/inn/en zum Studium nach Österreich kamen, eine deutliche Veränderung in Bezug auf ihren sozioökonomischen und kulturellen Background erfahren. Während die meisten der früheren Zuwander/er/innen und ersten

$93 \quad$ In den öffentlich zugänglichen Statistiken werden keine Angaben zum ethnolinguistischen oder religiösen Background der nach Österreich gekommenen Afghan/inn/en gemacht. 
afghanischen Fluchtmigrant/inn/en großteils der urbanen, oft westlich gebildeten Mittelund Oberschicht angehörten, entstammten viele der späteren Asylwerber/innen (v.a. ab der Mitte der 1990er Jahre) dem ländlichen Milieu Afghanistans. Sie verfügten oft über keine oder nur eine rudimentäre Schul- und Berufsausbildung. Unter ihnen waren und sind zahlreiche Personen (insbesondere in der Gruppe der UMF), die nicht direkt aus Afghanistan nach Österreich flohen, sondern zuvor schon längere Zeit in Pakistan oder Iran gelebt hatten oder sogar dort geboren worden waren. ${ }^{94}$

Auch was den Integrationsgrad in die österreichische Gesellschaft angeht, bestehen große Unterschiede. Die überwiegende Mehrheit der frühen afghanischen Zuwander/ er/innen (insbesondere jene aus der Zeit des Beginns der kriegerischen Handlungen in Afghanistan) sind längst österreichische Staatsbürger/innen, haben teilweise Österreicher/innen geheiratet, Familien gegründet und sind sehr gut in die österreichische Gesellschaft integriert. Viele von ihnen konnten sich als Ärzt/inn/e/n, Geschäftsleute und Pflegekräfte beruflich gut etablieren. Einige sind inzwischen auch schon in Pension. Schwieriger verlief die berufliche Integration gut ausgebildeter afghanischer Asylwerber/innen, die später nach Österreich kamen. Nur wenige konnten hier in ihren alten Berufen tätig sein und mussten oft einen beruflichen Abstieg in Kauf nehmen, um den Lebensunterhalt für sich und ihre Familien zu sichern. ${ }^{95}$ Sie legen oft einen erheblichen Wert auf die Ausbildung ihrer Kinder. Mittlerweile sind auch sie relativ gut in die österreichische Gesellschaft integriert.

Mit deutlich größeren Problemen sind jene Asylwerber/innen konfrontiert, die nur über einen geringen Bildungsstand verfügen und vor ihrem Ankommen in Österreich kaum Bezug zu westlichen Gesellschaften hatten. Ihnen sind viele der in Europa üblichen Verhaltensweisen oft fremd und es fällt ihnen anfänglich schwer, sich ohne entsprechende Unterstützung durch staatliche Stellen oder NGOs in einer für sie gänzlich neuen Umgebung zurechtzufinden. Zahlreiche Beispiele, v.a. aus der Gruppe der ursprünglichen UMF, belegen jedoch, dass mit entsprechender Unterstützung (z.B. durch Patenfamilien) selbst Personen mit nur minimaler oder keiner Schul- und Berufsausbildung einen erfolgreichen Berufseinstieg in Österreich schaffen können.

Obwohl in Österreich lebende Afghan/inn/en von zahlreichen österreichischen Politiker/inne/n und Mainstream-Medien oft als „nichtintegrierbare Problemgruppe“ dargestellt werden, die einem „archaischen“ Wertesystem anhängen würden, belegen die wenigen bislang vorliegenden Studien (vgl. KOHLBACHER et al. 2017), dass die hier lebenden Afghan/inn/en, genau wie andere Zuwander/er/innengruppen und alteingesessene Österreicher/innen in Bezug auf ihre Einstellungen und Werthaltungen sehr unterschiedliche Positionen vertreten.

94 Es liegen keine offiziellen Statistiken darüber vor, ob ein/e Asylwerber/in direkt aus Afghanistan oder aus dem Iran bzw. Pakistan nach Österreich gekommen ist. Anzumerken ist außerdem, dass erst seit ca. 2002 detaillierte Statistiken des Bundesministeriums für Inneres zugänglich sind.

95 Eine ähnliche Beobachtung konnte Kamran (2008, p. 95) für nach Deutschland geflohene Afghan/inn/en machen. 
Bezugnehmend auf den „Integrationsgrad“ der afghanischen Diaspora in Österreich können in Anlehnung an KAMRAN (2008, p. 96) drei Gruppen von „Diasporaafghan/ inn/en“ unterschieden werden:

1) Eine kleine Minderheit von Afghan/inn/en, die eine islamisch-afghanische Mikrokultur pflegt und kaum integriert ist.

2) Afghanische Migrant/inn/en, die vollständig in die Aufnahmegesellschaft integriert sind und nur sehr lose Bindungen zu Afghanistan haben.

3) Afghanen und Afghaninnen die zwar integriert sind, aber auch die afghanische Sprache und Kultur pflegen.

\subsubsection{Die transnationalen Kontakte der afghanischen Community in Österreich}

Bislang existieren keine detaillierten Studien, die Aufschluss über die transnationalen Kontakte der in Österreich lebenden Afghan/inn/en geben könnten. Sowohl aus den Respondent/inn/eninterviews der vorliegenden Studie wie auch aus früheren Erhebungen (z.B. Pilotstudie) ${ }^{96}$ sowie aus vereinzelten Hinweisen in anderen Untersuchungen (z.B. KAMRAN 2008) ergibt sich jedoch der Eindruck, dass viele der hier wohnhaften Afghan/inn/en enge verwandtschaftliche und/oder freundschaftliche Beziehungen $\mathrm{zu}$ Landsleuten in anderen europäischen Ländern (v.a. Deutschland, Niederlande, Schweden) oder in Nordamerika unterhalten. ${ }^{97}$ Auch zum ursprünglichen Herkunftsland (Afghanistan, Iran oder Pakistan) bestehen oft enge Kontakte. Mehrere unserer Respondent/inn/en haben noch Verwandte (z.B. Eltern, Geschwister etc.) im ursprünglichen Siedlungsgebiet. Einige warteten zum Zeitpunkt des Interviews auf die Einreise ihrer dort noch lebenden Ehefrau und/oder Kinder. Insgesamt 15 Personen unseres Samples kamen über Familienzusammenführung nach Österreich und mussten somit über einen längeren Zeitraum als transnationale Familien leben. In einzelnen Fällen war eine Zusammenführung der gesamten Familie auf Grund des Alters der Kinder, die inzwischen schon volljährig waren, nicht mehr möglich. In einem Fall entschied sich der Ehemann dafür, mit seinem Sohn in Afghanistan zu bleiben und nicht zu seiner vor ihm geflohenen Ehefrau zu ziehen, lediglich die noch minderjährigen Kinder kamen nach langem Warten zu ihrer Mutter nach Österreich.

Neben der Familienzusammenführung stellt die „Heiratsmigration“ eine weitere wichtige Form transnationaler Familienbeziehungen dar. Einzelne Respondent/inn/en, aber auch andere Afghan/inn/en haben eine Frau/einen Mann aus dem ursprünglichen Herkunftsgebiet geheiratet. Meist handelt es sich dabei um eine Person aus dem Umkreis der eigenen Verwandtschaftsgruppe. ${ }^{98}$

$96 \quad$ Bei den folgenden Ausführungen, wird in Ermangelung anderer Studien auf bisher vorliegende eigene Erhebungsdaten zurückgegriffen (vgl. u.a. KoHLBACHER und SCHIOCCET 2017).

97 Mehrere Respondent/inn/en verwiesen während der Erhebung auch darauf, dass ihr eigentliches Fluchtziel nicht Österreich, sondern Deutschland, Schweden etc. war, wo bereits Verwandte wohnten.

98 In Afghanistan sind Heiratsbeziehungen zwischen Verwandten sehr verbreitet, vgl. WAHAB et 
Einzelne Afghan/inn/en, vor allem jene, die über ein österreichisches Reisedokument verfügen, besuchen regelmäßig (z.B. Durchführung von Hilfsprojekten im Heimatland) oder zu speziellen Anlässen (z.B. Beerdigungen) die alte Heimat. Andere schicken, soweit es ihre ökonomische Lage erlaubt, Remittances an Verwandte in Afghanistan, Iran oder Pakistan. ${ }^{99}$ Die modernen Kommunikationsmittel Skype, WhatsApp und andere Internetdienste erlauben vielen in Österreich lebenden Afghan/inn/en eine rege Kontaktpflege mit ihren oft über die ganze Welt verstreuten Verwandten und Bekannten, auch in Afghanistan, wo mittlerweile Mobiltelefone eine weite Verbreitung gefunden haben.

\subsubsection{Afghanische Vereine in Österreich ${ }^{100}$}

Bedingt durch die geringe Zahl von in Österreich lebenden Afghan/inn/en kam es erst relativ spät zur Gründung eigenständiger afghanischer Vereine. Die wenigen vor Beginn der kriegerischen Auseinandersetzungen im Land studierenden oder arbeitenden Afghan/inn/en trafen sich - ihren persönlichen und/oder politischen Interessen entsprechend - in losen Zirkeln zum gemeinsamen Diskutieren oder Feiern von Festen.

Die mit der sowjetischen Invasion im Dezember 1979 einsetzende Fluchtmigration hunderttausender Afghan/inn/en und die Intensivierung der Kampfhandlungen in Afghanistan führten Anfang der 1980er Jahre zur Gründung zweier Vereine in Wien, in denen neben Österreicher/inne/n auch einige Afghan/inn/en aktiv waren. ${ }^{101}$ Zum einen das „Österreichische Hilfskomitee für Afghanistan“, das sich bis zur Einstellung seiner Aktivitäten 1994 vor allem um die medizinische und schulische Versorgung afghanischer Flüchtlinge in Pakistan kümmerte. Zum anderen das „Sozialistische Afghanistan Komitee“ (später umbenannt in Österreichisches Afghanistan Komitee), das durch Veranstaltungen und Publikationen auf die Situation in Afghanistan aufmerksam machen wollte. Da es in den 1980er Jahren noch keine entsprechenden Anlaufstellen für afghanische Asylwerber/innen gab, unterstützten beide Vereine einzelne Asylwerber/ innen bei den Asylverfahren und der Integration in die österreichische Gesellschaft. ${ }^{102}$

al. (2006).

99 Genaue Informationen bezüglich der Übermittlung von Remittances aus Österreich nach Afghanistan, Iran oder Pakistan sind nicht verfügbar.

100 Zu den afghanischen Vereinen in Österreich liegen bislang keine umfassenden Informationen vor. Einen kleinen Einblick liefert die Masterarbeit von AlAm aus dem Jahr 2008. In einigen Publikationen (z.B. AHMAD 2017, p. 39) sind Auflistungen von in Österreich existierenden Vereinen und religiösen Institutionen angeführt. Eigene Internetrecherchen, auch zu in anderen Veröffentlichungen genannten Vereinen, erbrachten nur geringe Informationen. In einem kürzlich veröffentlichten Bericht zum Diasporaengagement in Europa verweist AHмaD (2020) auf die Aktivitäten einiger afghanischer Vereine in Österreich.

101 Eigene Erinnerungen von Gabriele Rasuly-PALECZEK.

102 Zum Beispiel half das Sozialistische Afghanistan Komitee bei der Einrichtung von Alphabetisierungs- und Berufsausbildungskursen in Mödling und unterstützte zahlreiche Asylwerber/ innen, deren Asylanträge in erster Instanz abgelehnt worden waren, bei ihren Berufungen. 
Mit der steigenden Zahl von in Österreich lebenden Afghan/inn/en wurden ab den 1990er Jahren eine Reihe von afghanischen Vereinen und Organisationen gegründet. Die meisten hatten und haben ihren Sitz in Wien und fokussieren sich teilweise auf unterschiedliche Zielgruppen innerhalb der afghanischen Community (z.B. Frauen, UMF, afghanische Student/inn/en, Bevölkerung in Afghanistan). Die Ziele dieser Vereine decken ein breites Spektrum an Aktivitäten ab, wie z.B. Vernetzung der afghanischen Diaspora in Österreich, Förderung und Erhalt der afghanischen Kultur und Sprache (z.B. durch Dari-Sprachkurse für die zweite Generation, Konzerte und Dichterlesungen), Unterstützung von neu nach Österreich gekommenen Asylwerber/inne/n (z.B. durch Beratungstätigkeit, Anbieten von Deutschkursen), Verbreitung von Informationen über Afghanistan sowie Spendensammlungen für Projekte in Afghanistan, um damit einen Beitrag zum Wiederaufbau des Heimatlandes zu leisten.

Was die Intensität der Vereinsaktivitäten anbelangt, so dürfte diese sehr stark von den personellen und finanziellen Ressourcen der einzelnen Vereine abhängen. Die meisten Vereine sind ausschließlich auf ehrenamtliche Mitarbeiter/innen angewiesen und erhalten keine oder kaum Unterstützung durch österreichische NGOs oder öffentliche Stellen.

Wie viele Vereine es gibt, wie viele Mitglieder sie haben und wie aktiv sie tatsächlich sind, lässt sich nur schwer feststellen. Zahlreiche, in diversen Studien genannte Vereine (vgl. Alam 2008, p. 44; AHMAD 2017, p. 39; WALIZADEH 2017) konnten trotz Internetrecherche nicht eruiert werden bzw. dort, wo Informationen gefunden wurden, gab es wenige Hinweise auf die Vereinsgeschichte und Vereinsziele, die Mitgliederzahl etc. In einzelnen Fällen boten die Webseiten der Vereine Links zu Fotodokumentationen bereits abgehaltener Veranstaltungen (z.B. Nauroz-Feste oder Kulturabende).

Inwiefern die in Österreich bestehenden afghanischen Vereine transnational vernetzt sind (z.B. mit ähnlichen Vereinen in anderen europäischen Ländern oder weltweit), lässt sich nicht genau feststellen. Bislang scheint es so, als ob lediglich die Hazara-Community in Österreich dank der intensiven Bemühungen global agierender Hazara-Organisationen (z.B. Hazara World Council) transnational vernetzt ist. ${ }^{103}$

Neben den bereits genannten Vereinigungen existieren in Österreich, insbesondere in Wien, auch einige Gebetsräume und Moscheen, die von Afghan/inn/en besucht werden. ${ }^{104}$ In einer von WALIZADEH (2017) zusammengestellten Übersicht werden für Wien gerade einmal vier Gebetsräume genannt, darunter das „Islamische Zentrum“ im 21. Bezirk und das „Islamische Zentrum Imam Ali““ im 6. Bezirk. Insgesamt scheinen die in Österreich lebenden Afghan/inn/en Moscheevereinen eher reserviert gegenüberzustehen. Manche betrachten diese sogar als Gefahr für ihre Kinder. Viele unserer

103 In den letzten Jahren ist es den Hazara gelungen, sowohl innerhalb Afghanistans wie auch global durch Veranstaltungen, Webseiten etc. in Erscheinung zu treten (vgl. z.B. http://worldhazaracouncil.org/en/). Anderen afghanischen Gruppen, insbesondere ethnischen Minderheiten (z.B. Uzbeken), ist ein derartiger Mobilisierungsgrad bislang nicht geglückt.

104 Genauere Informationen zur Existenz afghanischer Moscheen oder Moscheevereine sowie zur Zahl der afghanischen Muslim/inn/e/n sunnitischen und schiitischen Glaubens konnten trotz intensiver Internetrecherche nicht eruiert werden. 
Respondent/inn/en erwähnten, dass sie eher zu Hause beten würden und nur anlässlich religiöser Feste oder eines Totengebetes eine Moschee aufsuchen würden.

Während zahlreiche andere Migrant/inn/engruppen in Österreich in speziellen Wirtschaftsbranchen tätig sind und dort oft schon eine Art von Monopol besitzen, haben sich spezielle „Ethnobusinesses“ der Afghan/inn/en noch nicht entwickelt. Einer der Hauptgründe dafür könnte in der geringen Finanzstärke afghanischer Zuwander/ er/innen nach Österreich liegen. Die meisten der in den letzten Jahren gekommenen Afghan/inn/en stammen eher aus der „unteren“ Mittelschicht und verfügen, nachdem sie ihre Flucht finanziert haben, meist über keine ausreichenden finanziellen Mittel, um in die Gründung eines eigenen Unternehmens zu investieren. Bislang gibt es nur zwei Sparten, in denen einige Afghan/inn/en in Österreich aktiv sind, einerseits in der Gastronomie (Gründung von afghanischen Restaurants) und andererseits im Importhandel (z.B. Eröffnung eines Nahrungsmittelgeschäfts, dessen Angebot sich primär an die eigenen Landsleute richtet, oder eines Teppichgeschäftes, in welchem auch andere kunsthandwerkliche Objekte, z.B. Schmuck, verkauft werden). 